\title{
THE COMMUTANT OF AN ANALYTIC TOEPLITZ OPERATOR
}

\author{
BY
}

\author{
CARL C. COWEN( $\left.{ }^{l}\right)$
}

\begin{abstract}
For a function $f$ in $H^{\infty}$ of the unit disk, the operator on $H^{2}$ of multiplication by $f$ will be denoted by $T_{f}$ and its commutant by $\left\{T_{f}\right\}^{\prime}$. For a finite Blaschke product $B$, a representation of an operator in $\left\{T_{B}\right\}^{\prime}$ as a function on the Riemann surface of $B^{-1} \circ B$ motivates work on more general functions. A theorem is proved which gives conditions on a family $\mathcal{F}$ of $H^{\infty}$ functions which imply that there is a function $h$ such that $\left\{T_{h}\right\}^{\prime}=\bigcap_{f \in \mathcal{F}}\left\{T_{f}\right\}^{\prime}$. As a special case of this theorem, we find that if the inner factor of $f-f(c)$ is a finite Blaschke product for some $c$ in the disk, then there is a finite Blaschke product $B$ with $\left\{T_{f}\right\}^{\prime}=\left\{T_{B}\right\}^{\prime}$. Necessary and sufficient conditions are given for an operator to commute with $T_{f}$ when $f$ is a covering map (in the sense of Riemann surfaces). If $f$ and $g$ are in $H^{\infty}$ and $f=h \circ g$, then $\left\{T_{f}\right\}^{\prime} \supset\left\{T_{g}\right\}^{\prime}$. This paper introduces a class of functions, the $H^{2}$-ancestral functions, for which the converse is true. If $f$ and $g$ are $H^{2}$-ancestral functions, then $\left\{T_{f}\right\}^{\prime} \neq\left\{T_{g}\right\}^{\prime}$ unless $f=h \circ g$ where $h$ is univalent. It is shown that inner functions and covering maps are $H^{2}$ ancestral functions, although these do not exhaust the class. Two theorems are proved, each giving conditions on a function $f$ which imply that $T_{f}$ does not commute with nonzero compact operators. It follows from one of these results that if $f$ is an $H^{2}$-ancestral function, then $T_{f}$ does not commute with any nonzero compact operators.
\end{abstract}

Introduction. In studying an operator on a Hilbert space, it is of interest to characterize the operators which commute with a given operator, for such a characterization should help in understanding the structure of the operator. Unless the operator is normal, very little is known about this general question, and even for subnormal operators the question seems to be difficult. This work tries to shed light on the problem for a special class of subnormal operators: the analytic Toeplitz operators. It is hoped that an understanding of these examples will point the way in the study of more general subnormal operators.

Received by the editors June 1, 1976.

AMS (MOS) subject classifications (1970). Primary 47B35, 47B20; Secondary 30A58, 30 A76.

Key words and phrases. Toeplitz operator, commutant, $H^{\infty}, H^{2}$, analytic function, inner function, universal covering map.

(')The research for this paper was undertaken in partial fulfillment of the requirements for the Ph.D. at the University of California at Berkeley under the supervision of Prof. Donald Sarason. I would like to thank Prof. Sarason for his help and inspiration throughout the research and writing. I would also like to thank Charles Stanton with whom I had several helpful discussions about Riemann surfaces. 
For a given function $f$ in $H^{\infty}$ of the unit disk $D$, the analytic Toeplitz operator with symbol $f$ is the operator on the Hardy space $H^{2}$ of multiplication by $f$. This operator will be denoted by $T_{f}$ and the set of operators which commute with it by $\left\{T_{f}\right\}^{\prime}$. We will try to characterize the operators in $\left\{T_{f}\right\}^{\prime}$ by using properties of $f$ considered as a function on the disk or as a function on the unit circle. The methods used are primarily function theoretic in character, so some of the results have interpretations for other operators which can be viewed as multiplication by an analytic function.

In their paper [11], Shields and Wallen consider commutants of operators which can be viewed as multiplication by $z$ in a Hilbert space of analytic functions. Later, it was noticed [4] that their methods can be used to show that if $f$ is univalent in $D$, then $\left\{T_{f}\right\}^{\prime}=\left\{T_{h} \mid h \in H^{\infty}\right\}$. Deddens and Wong [4] studied the problem using methods less related to function theory and raised six questions which have stimulated much of the further work on the problem. Based on this work, Baker, Deddens, and Ullman [3] proved that if $f$ is an entire function, then there is an integer $k$ such that $\left\{T_{f}\right\}^{\prime}=\left\{T_{z^{k}}\right\}^{\prime}$. Using function theoretic methods, Thomson [12]-[15] gave more general sufficient conditions that a commutant or intersection of commutants be equal to $\left\{T_{\phi}\right\}^{\prime}$ for a finite Blaschke product $\phi$.

One approach to the general problem is to identify a small class of functions such that $\left\{T_{\phi}\right\}^{\prime}$ is well understood when $\phi$ is one of these functions, and moreover, for each $f$ in $H^{\infty}$, there is a function $\phi$ in the special class with $\left\{T_{f}\right\}^{\prime}=\left\{T_{\phi}\right\}^{\prime}$. The spirit of several of the questions raised by Deddens and Wong seems to be "Is the class of inner functions such a class?" Abrahamse [1] and Abrahamse and Ball [2], using results about bundle shifts, have shown that the class of inner functions is not broad enough to accomplish this. It is hoped that this paper will help identify such a class.

In $\$ 1$, basic theorems and concepts are presented. The notion of an ancestral function is introduced, and it is shown that the commutant of a Toeplitz operator whose symbol is ancestral behaves well with respect to composition of the symbol. It is shown that inner functions are ancestral.

$\$ 2$ describes the commutant of a Toeplitz operator whose symbol is a finite Blaschke product. Although such commutants are well known, the description in this section is not well known. The techniques used in the description are motivation for the work of $\$ \S 3$ and 4.

$\$ 3$ presents sufficient conditions for the intersection of commutants of analytic Toeplitz operators to be the commutant of a single analytic Toeplitz operator. Special cases of this result give conditions on a function $h$ so that $\left\{T_{h}\right\}^{\prime}=\left\{T_{\psi}\right\}^{\prime}$ where $\psi$ has a special form, for example, where $\psi$ is a finite Blaschke product.

$\$ 4$ gives necessary and sufficient conditions for an operator to commute 
with $T_{\phi}$ if $\phi$ is a covering map. It is shown that a covering map is an ancestral function. The examples of Abrahamse [1] and Abrahamse and Ball [2] arise from a covering map.

Deddens and Wong [4] raise the question "If $f$ is a nonconstant $H^{\infty}$ function, can $T_{f}$ commute with a nonzero compact operator?” In $\$ 5$ we prove two theorems, each of which gives conditions on $f$ which imply that $T_{f}$ does not commute with nonzero compact operators. In particular, we show that this is the case if $f$ is an ancestral function.

1. General theory. In this section we identify necessary and sufficient conditions for an operator to be in the commutant of an analytic Toeplitz operator and draw conclusions about relations between commutants.

The kernels for evaluating functions at points of the disk play a key role because they are eigenvectors for the adjoints of analytic Toeplitz operators.

Definition. For $\alpha$ in $D$, the kernel function at $\alpha$, denoted $K_{\alpha}$, is the function $K_{\alpha}(z)=(1-\bar{\alpha} z)^{-1}$.

Cauchy's theorem implies that for $h$ in $H^{2}$ we have $\left\langle h, K_{\alpha}\right\rangle=h(\alpha)$, where $\langle\cdot, \cdot\rangle$ is the inner product in $H^{2}$.

The following lemma, which has been used in various forms by other authors [3], [4], [11] [15], is the foundation for much of this work.

Fundamental Lemma. For $S$ in $\mathscr{B}\left(H^{2}\right)$ and $f$ in $H^{\infty}$ the following are equivalent.

(1) $S$ commutes with $T_{f}$.

(2) For all $\alpha$ in $D, S^{*} K_{\alpha} \perp(f-f(\alpha)) H^{2}$.

(3) There is a set $J \subset D$ such that $\Sigma_{\alpha \in J}(1-|\alpha|)=\infty$ and for all $\alpha$ in $J$, $S^{*} K_{\alpha} \perp(f-f(\alpha)) H^{2}$.

Proof. (1) $\Rightarrow(2)$ Let $S$ be in $\left\{T_{f}\right\}^{\prime}$. For all $g$ in $H^{2}$ and $\alpha$ in $D$, we have

$$
\begin{aligned}
& \left\langle(f-f(\alpha)) g, S^{*} K_{\alpha}\right\rangle=\left\langle S T_{f} g, K_{\alpha}\right\rangle-f(\alpha)\left\langle S g, K_{\alpha}\right\rangle \\
& \quad=\left\langle T_{f} S g, K_{\alpha}\right\rangle-f(\alpha)\left\langle S g, K_{\alpha}\right\rangle=f(\alpha)(S g)(\alpha)-f(\alpha)(S g)(\alpha)=0 .
\end{aligned}
$$

(2) $\Rightarrow$ (3) Let $J=D$.

(3) $\Rightarrow$ (1) Let $S$ be in $\mathscr{B}\left(H^{2}\right)$ with $S^{*} K_{\alpha} \perp(f-f(\alpha)) H^{2}$ for $\alpha$ in $J$. For $g$ in $H^{2}$ and $\alpha$ in $J$ we have

$$
\begin{aligned}
0 & =\left\langle(f-f(\alpha)) g, S^{*} K_{\alpha}\right\rangle=\left(S T_{f} g\right)(\alpha)-f(\alpha)(S g)(\alpha) \\
& =\left(S T_{f} g\right)(\alpha)-\left(T_{f} S g\right)(\alpha) .
\end{aligned}
$$

Since $J$ is not a Blaschke sequence, this means $S T_{f} g=T_{f} S g$, hence $S T_{f}=$ $T$ f .

Corollary. If $f$ is in $H^{\infty}$ and $g$ is bounded and analytic on $f(D)$, then $\left\{T_{f}\right\}^{\prime} \subset\left\{T_{g \circ f}\right\}^{\prime}$. 
Proof. Let $\beta$ be in $f(D)$. Since $(g(z)-g(\beta)) /(z-\beta)$ is bounded and analytic on $f(D),(g \circ f-g(\beta)) /(f-\beta)$ is in $H^{\infty}$. For all $\alpha$ in $D$, we have

$$
g \circ f-g(f(\alpha))=\frac{g \circ f-g(f(\alpha))}{f-f(\alpha)}(f-f(\alpha)) \text {, }
$$

so that

$$
(g \circ f-g(f(\alpha))) H^{2} \subset(f-f(\alpha)) H^{2} .
$$

If $S$ is in $\left\{T_{f}\right\}^{\prime}$, then for all $\alpha$ in $D, S^{*} K_{\alpha} \perp(f-f(\alpha)) H^{2}$, hence $S^{*} K_{\alpha} \perp(g \circ f-g(f(\alpha))) H^{2}$, and we have $S \in\left\{T_{g \circ f}\right\}^{\prime}$.

COROLlaRY. If $f$ is in $H^{\infty}$ and $\phi$ is bounded, univalent, and analytic on $f(D)$, then $\left\{T_{f}\right\}^{\prime}=\left\{T_{\phi \circ f}\right\}^{\prime}$.

From these corollaries we see that the situation for commutants is considerably different from that for invariant subspaces. For example, if $\phi$ is a conformal map of the disk onto the disk slit along $[0,1)$, then $\left\{T_{\phi}\right\}^{\prime}=$ $\left\{T_{z}\right\}^{\prime}$ but there are subspaces invariant for $T_{\phi}$ which are not invariant for $T_{z}$. (The subspace spanned by $\left\{1, \phi, \phi^{2}, \ldots\right\}$ is obviously invariant for $T_{\phi}$, but since $\phi$ maps two arcs of $\partial D$ onto the interval $[0,1]$, the subspace does not contain $T_{z}$ 1.)

Easy examples show that, in general, the converse of the first corollary is false, so a function for which the converse is true must be special in some way. This feeling and the statement of the lemma suggest the following definition. (If $\mathfrak{V}$ is a set of vectors, we let $\bigvee \mathfrak{V}$ be the closed subspace spanned by $\mathcal{T}$.)

Definition. For $f$ in $H^{\infty}$, we say $f$ is $H^{2}$-ancestral if for every $\alpha$ in $D$, we have

$$
\bigvee\left\{S^{*} K_{\alpha} \mid S \in\left\{T_{f}\right\}^{\prime}\right\}=\left[(f-f(\alpha)) H^{2}\right]^{\perp}
$$

Since we will only be considering the Hilbert space $H^{2}$, we will refer to such an $f$ as ancestral. We observe that $\left\{T_{h}\right\}^{\prime} \supset\left\{T_{f}\right\}^{\prime}$ if and only if $T_{h}$ is in $\left\{T_{f}\right\}^{\prime \prime}$, so the following theorem is a statement about double commutants.

THEOREM 1. If $f$ and $h$ are in $H^{\infty}, f$ is ancestral, and $\left\{T_{f}\right\}^{\prime} \subset\left\{T_{h}\right\}^{\prime}$, then there is a function $g$ analytic on $f(D)$ with $h=g \circ f$.

Proof. For each $\alpha$ in $D$ we have

$$
\begin{aligned}
{\left[(f-f(\alpha)) H^{2}\right]^{\perp} } & =\bigvee\left\{S^{*} K_{\alpha} \mid S \in\left\{T_{f}\right\}^{\prime}\right\} \subset \bigvee\left\{S^{*} K_{\alpha} \mid S \in\left\{T_{h}\right\}^{\prime}\right\} \\
& \subset\left[(h-h(\alpha)) H^{2}\right]^{\perp} .
\end{aligned}
$$

For $w$ in $f(D)$, choose $\alpha$ in $D$ with $f(\alpha)=w$ and let $g(w)=h(\alpha)$. The function $g$ is well defined, for if $f(\alpha)=f(\beta)$, then $K_{\beta} \in\left[(f-f(\alpha)) H^{2}\right]^{\perp} \subset$ 
$\left[(h-h(\alpha)) H^{2}\right]^{\perp}$ so $h(\alpha)=h(\beta)$ also. Moreover, $g$ is analytic. Indeed, if $w=f(\alpha), f^{\prime}(\alpha) \neq 0$, and if $\zeta$ is the branch of $f^{-1}$ defined in a neighborhood of $w$ with $\zeta(w)=\alpha$, then in this neighborhood $g=h \circ \zeta$. Since $g$ is single valued, it follows immediately that $g$ is analytic on all of $f(D)$ and $h=g \circ f$.

COROLLARY. If $f_{1}$ and $f_{2}$ are ancestral $H^{\infty}$ functions and $\left\{T_{f_{1}}\right\}^{\prime}=\left\{T_{f_{2}}\right\}^{\prime}$, then there is a univalent function $\phi$ so that $f_{2}=\phi \circ f_{1}$.

Proof. Applying Theorem 1 twice, we see that there are functions $\phi$ and $\psi$ so that $f_{2}=\phi \circ f_{1}$ and $f_{1}=\psi \circ f_{2}$. Therefore $f_{1}=(\psi \circ \phi) \circ f_{1}$, which means $\psi \circ \phi$ is the identity on $f_{1}(D)$, so $\phi$ is univalent.

The corollary shows that if two ancestral functions are not each composites of the other, then the commutants of their Toeplitz operators are different. Thus, the following theorem provides a large (and well-known) collection of different commutants. We will show in $\$ 4$ that not all ancestral functions are inner functions.

THEOREM 2. If $\phi$ is an inner function, then $\phi$ is ancestral.

Proof. Let $\alpha$ be in $D$, let $M=\left[(\phi-\phi(\alpha)) H^{2}\right]^{\perp}$, and let $v$ be a vector in $M$. We will find $S$ in $\left\{T_{\phi}\right\}^{\prime}$ so that $S^{*} K_{\alpha}=v$.

Let

$$
\psi(z)=(\phi(z)-\phi(\alpha))(1-\overline{\phi(\alpha)} \phi(z))^{-1} .
$$

The function $\psi$ is also an inner function, and because the function

$$
w \rightarrow(w-\phi(\alpha))(1-\overline{\phi(\alpha)} w)^{-1}
$$

is univalent, $\left\{T_{\phi}\right\}^{\prime}=\left\{T_{\psi}\right\}^{\prime}$.

Now $M=H^{2} \ominus \psi H^{2}$, so $H^{2}=\bigoplus_{k=0}^{\infty} \psi^{k} M$. Let $\tilde{S}$ be a bounded operator on $M$ such that $\tilde{S}^{*} K_{\alpha}=v$. We define $S$ on $H^{2}$ by

$$
S\left(\sum_{k=0}^{\infty} w_{k} \psi^{k}\right)=\sum_{k=0}^{\infty}\left(\tilde{S} w_{k}\right) \psi^{k}
$$

where $w_{k} \in M$ for each $k$ and $\Sigma_{k=0}^{\infty}\left\|w_{k}\right\|^{2}<\infty$. Easy calculations show that $S$ is bounded $(\|S\|=\|\tilde{S}\|), S$ commutes with $T_{\psi}$ (hence with $T_{\phi}$ ), and $S^{*} K_{\alpha}=v$.

2. An example. In this section we will describe the commutant of a Toeplitz operator whose symbol is a finite Blaschke product. A matrix representation for an operator in such a commutant is well known. On the other hand, the class of Toeplitz operators for which this sort of representation can be derived seems to be limited to those whose symbol is an inner function. We will describe the commutant in terms, based on the fundamental lemma, 
more easily generalized. This way of looking at the commutant was suggested by the work of James Thomson [12], [13].

Throughout this section, $\phi$ will be a Blaschke product with $n$ zeroes, $1 \leqslant n<\infty$. We begin by showing that an operator which commutes with $T_{\phi}$ must map bounded functions to bounded functions, and we obtain an estimate for the norm of the operator considered as a mapping from $H^{\infty}$ to $H^{\infty}$.

Lemma. Let $S$ be a bounded operator on $H^{2}$ and let $f$ be in $H^{\infty}$. If $\alpha$ is in $D, \Phi$ is inner, and $S^{*} K_{\alpha} \perp \Phi H^{2}$ then

$$
|(S f)(\alpha)| \leqslant\|S\|\|f\|_{\infty} \sqrt{\left(1-|\Phi(0)|^{2}\right) /\left(1-|\alpha|^{2}\right)} .
$$

Proof.

$$
\begin{aligned}
& |(S f)(\alpha)|=\left|\left\langle S f, K_{\alpha}\right\rangle\right|=\left|\left\langle S((1-\overline{\Phi(0)} \Phi) f), K_{\alpha}\right\rangle\right| \\
& \quad\left\langle\|S\|\|(1-\overline{\Phi(0)} \Phi) f\|_{2}\left\|K_{\alpha}\right\|_{2} \leqslant\|S\|\|f\|_{\infty} \sqrt{\left(1-|\Phi(0)|^{2}\right) /\left(1-|\alpha|^{2}\right)} .\right.
\end{aligned}
$$

Proposition. If $\phi$ is a finite Blaschke product and $S$ is in $\left\{T_{\phi}\right\}^{\prime}$, then for all $f$ in $H^{\infty}$, Sf is in $H^{\infty}$ and

$$
\|S f\|_{\infty} \leqslant\|S\| \sqrt{(1+|\phi(0)|) /(1-|\phi(0)|)} \sqrt{\left\|\phi^{\prime}\right\|_{\infty}}\|f\|_{\infty} .
$$

Proof. Without loss of generality, we may assume $\phi(0)=0$, for $\psi=(\phi-\phi(0))(1-\overline{\phi(0)} \phi)^{-1}$ is a finite Blaschke product, $\psi(0)=0,\left\{T_{\psi}\right\}^{\prime}=$ $\left\{T_{\phi}\right\}^{\prime}$, and

$$
\left\|\psi^{\prime}\right\|_{\infty} \leqslant(1+|\phi(0)|)(1-|\phi(0)|)^{-1}\left\|\phi^{\prime}\right\|_{\infty} .
$$

By the fundamental lemma, for every $\alpha$ in $D$ we have

$$
S^{*} K_{\alpha} \perp(\phi-\phi(\alpha)) H^{2} .
$$

Since $(\phi-\phi(\alpha)) H^{2}=(\phi-\phi(\alpha))(1-\overline{\phi(\alpha)} \phi)^{-1} H^{2}$, the above lemma shows that

$$
\begin{aligned}
|(S f)(\alpha)| & <\|S\|\|f\|_{\infty} \sqrt{\left[1-\left|\frac{\phi(0)-\phi(\alpha)}{1-\overline{\phi(\alpha)} \phi(0)}\right|^{2}\right]\left[1-|\alpha|^{2}\right]^{-1}} \\
& =\|S\|\|f\|_{\infty} \sqrt{\frac{1-|\phi(\alpha)|^{2}}{1-|\alpha|^{2}}}
\end{aligned}
$$

Now 


$$
\lim _{r \rightarrow 1} \frac{1-\left|\phi\left(r e^{i \theta}\right)\right|}{1-\left|r e^{i \theta}\right|} \leqslant \lim _{r \rightarrow 1}\left|\frac{\phi\left(e^{i \theta}\right)-\phi\left(r e^{i \theta}\right)}{e^{i \theta}-r e^{i \theta}}\right|=\left|\phi^{\prime}\left(e^{i \theta}\right)\right| .
$$

So

$$
\lim _{r \rightarrow 1}\left|(S f)\left(r e^{i \theta}\right)\right| \leqslant\|S\|\|f\|_{\infty} \sqrt{\left|\phi^{\prime}\left(e^{i \theta}\right)\right|} .
$$

Since $\phi^{\prime}$ is bounded on $\partial D$, this shows $S f$ is in $H^{\infty}$ and

$$
\|S f\|_{\infty} \leqslant\|S\| \sqrt{\left\|\phi^{\prime}\right\|_{\infty}}\|f\|_{\infty} .
$$

It would be interesting to know whether the converse to this proposition is true; that is, if $g$ is in $H^{\infty}$ and every operator in $\left\{T_{g}\right\}^{\prime}$ maps bounded functions to bounded functions, does $\left\{T_{g}\right\}^{\prime}=\left\{T_{\phi}\right\}^{\prime}$ for some finite Blaschke product $\phi$ ? If $g$ is an infinite inner function, it is easy to find operators in $\left\{T_{g}\right\}^{\prime}$ which do not map bounded functions to bounded functions.

The usual representation for $S$ in $\left\{T_{\phi}\right\}^{\prime}$ comes from the decomrosition $H^{2}=\bigoplus_{k=0}^{\infty} \phi^{k}\left(H^{2} \ominus \phi H^{2}\right)$. If $\psi_{1}, \ldots, \psi_{n}$ is a basis for $H^{2} \ominus \phi H^{2}$, then $\psi_{1}, \ldots, \psi_{n}, \phi \psi_{1}, \ldots, \phi \psi_{n}, \phi^{2} \psi_{1}, \ldots$ is a basis for $H^{2}$, and with respect to this basis the matrix for $S$ has the block form

$$
\left(\begin{array}{ccccc}
A_{0} & 0 & 0 & 0 & \cdots \\
A_{1} & A_{0} & 0 & 0 & \cdots \\
A_{2} & A_{1} & A_{0} & 0 & \cdots \\
A_{3} & A_{2} & A_{1} & A_{0} & \cdots \\
\vdots & \vdots & \vdots & \vdots &
\end{array}\right)
$$

where each $A_{j}$ is an $n$ by $n$ matrix. On each of the subspaces $\left[\psi_{j}, \psi_{j} \phi, \psi_{j} \phi^{2}, \psi_{j} \phi^{3}, \ldots\right], S$ acts by multiplication: if $g=\Sigma a_{k} \phi^{k}$, then $S\left(g \psi_{j}\right)$ $=g S\left(\psi_{j}\right)$. Since $\psi_{j}$ is in $H^{\infty}$, it follows from the proposition above that $S \psi_{j}$ is in $H^{\infty}$. Thus in this point of view, an operator in $\left\{T_{\phi}\right\}^{\prime}$ is determined by $n$ $H^{\infty}$ functions, which act by multiplication as above, and whose coefficients with respect to the above basis comprise the columns of the matrix for the operator.

A finite Blaschke product with $n$ zeroes is an $n$ to 1 conformal map of $\bar{D}$ onto $\bar{D}$, and it is easy to show that its derivative has exactly $n-1$ zeroes in $D$ and none on $\partial D$. If we let $F=\left\{\beta \in D \mid\right.$ there is $\alpha$ in $D, \phi^{\prime}(\alpha)=0$ and $\phi(\alpha)=\phi(\beta)\}$, then $F$ is a finite set, and $\phi^{-1} \circ \phi$ is an $n$-branched analytic function defined and arbitrarily continuable in $D \backslash F$. Not all of the branches of $\phi^{-1} \circ \phi$ can be continued to a different branch, for example $z$ is a single valued branch of $\phi^{-1} \circ \phi$. The Riemann surface for $\phi^{-1} \circ \phi$ over $D$ is an $n$-sheeted cover of $D$ with at most $n(n-1)$ branch points, and is not connected. We will denote this Riemann surface by $W$, and a point of $W$ 
C. C. COWEN

lying over $D \backslash F$ by $(\eta, \alpha)$, where $\alpha$ is in $D \backslash F$ and $\eta$ is a branch of $\phi^{-1} \circ \phi$ defined in a neighborhood of $\alpha$.

TheOREM 3. Let $\phi, F$, and $W$ be as above. If $S$ is in $\left\{T_{\phi}\right\}^{\prime}$, then there is a function, $G$, bounded and analytic on $W$, so that for $h$ in $H^{2}$ and $\alpha$ in $D \backslash F$ we have

$$
(S h)(\alpha)=\left(\phi^{\prime}(\alpha)\right)^{-1} \sum G((\eta, \alpha)) \eta^{\prime}(\alpha) h(\eta(\alpha))
$$

where the sum is taken over the $n$ branches of $\phi^{-1} \circ \phi$ at $\alpha$. Moreover, if $\alpha_{0}$ is a zero of order $m$ of $\phi^{\prime}$, and $\psi_{1}, \ldots, \psi_{n}$ is a basis of $\left[\left(\phi-\phi\left(\alpha_{0}\right)\right) H^{2}\right]^{\perp}$, then $G$ has the property that

$$
\sum G((\eta, \alpha)) \eta^{\prime}(\alpha) \psi_{l}(\eta(\alpha)) \text { has a zero of order } m \text { at } \alpha_{0}
$$

for $l=1,2, \ldots, n$.

Conversely, if $G$ is a bounded analytic function on $W$ which has the properties $(* *)$ at each zero of $\phi^{\prime}$, then (*) defines a bounded linear operator $S$, on $H^{2}$, and $S$ is in $\left\{T_{\phi}\right\}^{\prime}$.

Proof. Let $S$ be in $\left\{T_{\phi}\right\}^{\prime}$. For $z$ and $\beta$ in $D$, we have

$$
\left|\frac{\phi(z)-\phi(\beta)}{z-\beta}\right|=\left|\frac{1}{z-\beta} \int_{\beta}^{z} \phi^{\prime}(\tau) d \tau\right| \leqslant \sup \left\{\mid \phi^{\prime}(\tau) \| \tau \text { is in }[\beta, z]\right\} .
$$

So for each $\beta$ in $D$, we have $\|(\phi-\phi(\beta)) /(z-\beta)\|_{\infty} \leqslant\left\|\phi^{\prime}\right\|_{\infty}$. Thus it follows from the proposition above that there is a constant $M$ so that if $\alpha$ is in $D$ and $\eta$ is a branch of $\phi^{-1} \circ \phi$ at $\alpha$, then

$$
\left|S\left(\frac{\phi-\phi(\alpha)}{z-\eta(\alpha)}\right)(\alpha)\right| \leqslant\left\|S\left(\frac{\phi-\phi(\alpha)}{z-\eta(\alpha)}\right)\right\|_{\infty}<M .
$$

We define $G$ on $W$ by

$$
G((\eta, \alpha))=S\left(\frac{\phi-\phi(\alpha)}{z-\eta(\alpha)}\right)(\alpha)
$$

so $G$ is in $H^{\infty}(W)$.

If $\alpha_{1}$ is in $D \backslash F$ and $\eta_{1}, \ldots, \eta_{n}$ are the branches of $\phi^{-1} \circ \phi$ defined near $\alpha_{1}$, then for $\alpha$ near $\alpha_{1}$, the subspace $\left[(\phi-\phi(\alpha)) H^{2}\right]^{\perp}$ is spanned by $K_{\eta_{1}(\alpha)}, \ldots, K_{\eta_{n}(\alpha)}$. The fundamental lemma shows that there are functions $c_{1}, \ldots, c_{n}$ defined in a neighborhood of $\alpha_{1}$ so that

$$
S^{*} K_{\alpha}=\sum_{j=1}^{n} \overline{c_{j}(\alpha)} K_{\eta(\alpha)}
$$

Now 


$$
S\left(\frac{\phi-\phi(\alpha)}{z-\eta_{j}(\alpha)}\right)(\alpha)=\left\langle\frac{\phi-\phi(\alpha)}{z-\eta_{j}(\alpha)}, S^{*} K_{\alpha}\right\rangle=c_{j}(\alpha) \phi^{\prime}\left(\eta_{j}(\alpha)\right),
$$

so that $c_{j}(\alpha)=G\left(\left(\eta_{j}, \alpha\right)\right) / \phi^{\prime}\left(\eta_{j}(\alpha)\right)$. From the equality

$$
S^{*} K_{\alpha}=\sum_{j=1}^{n} \frac{\overline{G\left(\left(\eta_{j}, \alpha\right)\right)}}{\phi^{\prime}\left(\eta_{j}(\alpha)\right)} K_{\eta(\alpha)},
$$

it follows that for every $h$ in $H^{2}$,

$$
(S h)(\alpha)=\sum_{j=1}^{n} \frac{\overline{G\left(\left(\eta_{j}, \alpha\right)\right)}}{\phi^{\prime}\left(\eta_{j}(\alpha)\right)} h\left(\eta_{j}(\alpha)\right) .
$$

Since $\phi(\eta(\alpha))=\phi(\alpha)$, we have $\phi^{\prime}(\eta(\alpha)) \eta^{\prime}(\alpha)=\phi^{\prime}(\alpha)$ and the representation (*) follows. The properties (**) follow trivially from the representation (*) and the observation that the functions $S \psi_{l}$ do not have poles in the disk.

Conversely, if $G$ is in $H^{\infty}(W)$, since $\phi(\eta(\alpha))=\phi(\alpha)$, the operator defined by (*) commutes formally with $T_{\phi}$. It is also clear from the boundedness of $G$ on $W$, and the boundedness of $\left(\phi^{\prime}(\alpha)\right)^{-1}$ and $\eta^{\prime}(\alpha)$ near $\partial D$, that there is a constant $C$ such that

$$
\frac{1}{2 \pi} \int\left|(S h)\left(r e^{i t}\right)\right|^{2} d t \leqslant C\|h\|_{2}^{2}
$$

for $r$ near 1. Thus, to conclude $S$ is a bounded operator which commutes with $T_{\phi}$, we need only show $S h$ is analytic in $D$ for each $h$ in $H^{2}$. The analyticity is clear for points $\alpha$ such that $\phi^{\prime}(\alpha) \neq 0$. If $\phi^{\prime}\left(\alpha_{0}\right)=0$, the conditions (**) guarantee that $S h$ does not have a pole at $\alpha_{0}$ if $h \perp\left(\phi-\phi\left(\alpha_{0}\right)\right) H^{2}$, so we consider $h=\left(\phi-\phi\left(\alpha_{0}\right)\right) g$. In this case we have

$$
\begin{aligned}
(S h)(\alpha) & =\left(\phi^{\prime}(\alpha)\right)^{-1} \sum G((\eta, \alpha)) \eta^{\prime}(\alpha)\left(\phi(\eta(\alpha))-\phi\left(\alpha_{0}\right)\right) g(\eta(\alpha)) \\
& =\sum G((\eta, \alpha)) g(\eta(\alpha)) \frac{\phi(\eta(\alpha))-\phi\left(\alpha_{0}\right)}{\phi^{\prime}(\eta(\alpha))} .
\end{aligned}
$$

Since

$$
\lim _{\alpha \rightarrow \alpha_{0}} \frac{\phi(\eta(\alpha))-\phi\left(\alpha_{0}\right)}{\phi^{\prime}(\eta(\alpha))}=0
$$

for each branch $\eta$ of $\phi^{-1} \circ \phi, \lim _{\alpha \rightarrow \alpha_{0}}(S h)(\alpha)=0$.

Each of the conditions (**) on functions $G$ in $H^{\infty}(W)$ is that $G$ be in the kernel of a bounded linear functional which can be expressed as the composition of a map from $H^{\infty}(W)$ to $H^{\infty}(W)$, a map of $H^{\infty}(W)$ to $H^{\infty}(D)$, and the evaluation of a derivative at a point of $D$. The first of these is a multiplication: given $\psi$ in $H^{\infty}(D)$, we define $\Psi: H^{\infty}(W) \rightarrow H^{\infty}(W)$ by

$$
(\Psi G)(\eta, \alpha)=\psi(\eta(\alpha)) G((\eta, \alpha)) .
$$


The second is the map $\pi: H^{\infty}(W) \rightarrow H^{\infty}(D)$ given by

$$
(\pi G)(\alpha)=\sum G((\eta, \alpha)) \eta^{\prime}(\alpha) .
$$

The proof that the function $\pi G$ is bounded involves finding local coordinates for $W$ at branch points, writing $G$ and $\eta$ in terms of these coordinates, and observing that the singularities arising from the $\eta^{\prime}$ terms sum to zero. Thus $\left\{T_{\phi}\right\}^{\prime}$ is identified with a subspace of $H^{\infty}(W)$ of finite codimension, and the functions in $H^{\infty}(W)$ act by multiplication as in (*).

To make these ideas clearer, we consider the explicit example $\phi(z)=z^{3}$. Let $\lambda$ be a primitive cube root of 1 . The three branches of $\phi^{-1} \circ \phi$ are each single valued: $\eta_{0}(z)=z, \eta_{1}(z)=\lambda z$, and $\eta_{2}(z)=\lambda^{2} z$. The Riemann surface $W$ consists of three disjoint disks, so a function $G$ in $H^{\infty}(W)$ consists of three functions $g_{0}, g_{1}, g_{2}$ in $H^{\infty}(D): G\left(\left(\eta_{j}, \alpha\right)\right)=g_{j}(\alpha)$. The operator $S$ associated with this $G$ is defined by

$$
(S h)(\alpha)=\left(3 \alpha^{2}\right)^{-1} \sum_{j=0}^{2} \lambda^{j} g_{j}(\alpha) h\left(\lambda^{j} \alpha\right) .
$$

Since $\phi^{\prime}$ has a zero of order 2 at 0 and $1, z, z^{2} \operatorname{span}\left[(\phi-\phi(0)) H^{2}\right]^{\perp}$, there are six conditions $(* *)$, three of which are nontrivial:

(i) $\sum_{j=0}^{2} \lambda^{j} g_{j}(0)=0$,

(ii) $\sum_{j=0}^{2} \lambda^{j} g_{j}^{\prime}(0)=0$, and

(iii) $\sum_{j=0}^{2} \lambda^{2 j} g_{j}(0)=0$.

In $\S \S 3$ and 4 , the commutant of a Toeplitz operator $T_{f}$ for more general functions $f$ will be developed in terms of the branches of $f^{-1} \circ f$. This development is fairly easy for a finite Blaschke product $\phi$ because there are only finitely many branches of $\phi^{-1} \circ \phi$ and each of these is continuable in $D \backslash F$ where $F$ is a finite set. For more general functions $f$, there may be infinitely many branches of $f^{-1} \circ f$ and some of these branches may not be continuable in a large subset of $D$. $\$ 3$ deals with a special case in which only finitely many branches are important, and $\$ 4$ deals with a special case in which each branch is arbitrarily continuable in $D$, although there are infinitely many branches.

3. The aim of this section is to prove a theorem on the intersection of commutants of analytic Toeplitz operators whose symbols are composites of a fixed function and which satisfy a finiteness condition. A special case of this theorem establishes a connection between $\left\{T_{h}\right\}^{\prime}$ and $\left\{T_{f}\right\}^{\prime}$ when $h$ is a composite of $f$ and satisfies a finiteness condition. Another special case gives a sufficient condition for $T_{h}$ to have the same commutant as $T_{\phi}$ for some finite Blaschke product $\phi$. This theorem and its corollaries generalize results of Deddens and Wong [4], Baker, Deddens, and Ullman [3], and Thomson 
[13], [14], [15]. The proof techniques are extensions of those used by these authors, especially Thomson.

When $h$ is in $H^{\infty}$, we write $\operatorname{Inn}(h)$ for the inner factor of $h$. We begin by stating the theorem.

THEOREM 4. Let $f$ be in $H^{\infty}$ with $f(D)=\Omega$, and let $\mathscr{F}$ be a family of nonconstant functions such that for each $h$ in $\mathscr{F}$, there is $g$ in $H^{\infty}(\Omega)$ with $h=g \circ f$. If there are points $\alpha_{1}, \alpha_{2}, \ldots, \alpha_{n}$ in $D$ (not necessarily distinct) so the greatest common divisor of $\left\{\operatorname{Inn}\left(h-h\left(\alpha_{1}\right)\right) \mid h \in \mathscr{F}\right\}$ is $\prod_{j=1}^{n} \operatorname{Inn}\left(f-f\left(\alpha_{j}\right)\right)$, then there is a function $\psi$ in $H^{\infty}(\Omega)$ so that $\cap_{h \in \mathcal{F}}\left\{T_{h}\right\}^{\prime}=\left\{T_{\psi \circ f}\right\}^{\prime}$.

In the previous section, the commutant of $T_{\phi}$ for $\phi$ a finite Blaschke product was described in terms of the branches of $\phi^{-1} \circ \phi$. If $h=g \circ \phi$ then every branch of $\phi^{-1} \circ \phi$ is also a branch of $h^{-1} \circ h$. Thus if we wish to show that $\left\{T_{h}\right\}^{\prime}=\left\{T_{\phi}\right\}^{\prime}$ we might try to find the appropriate branches of $h^{-1} \circ h$ and use them to reconstruct $\phi$. In the case considered by Theorem 4 , the identity function $z: D \rightarrow D$ is replaced by the function $f: D \rightarrow \Omega$ and the role of the finite Blaschke product $\phi$ is played by the function $\psi$, which we will see has certain finiteness properties analogous to those of a finite Blaschke product.

We will first prove a lemma concerning coanalytic functions from plane domains into $H^{2}$. (A function will be called coanalytic if it is given locally by a power series in $\bar{z}_{\text {. }}$ ) The first step in the proof of the theorem is to use the lemma and the condition at $\alpha_{1}$ to find a representation for operators in the commutant which is valid in a neighborhood of $\alpha_{1}$. We can apply the lemma because the function $\alpha \rightarrow S^{*} K_{\alpha}$ is coanalytic. This representation will involve some of the branches of $g^{-1} \circ g$, where $g \circ f$ is in $\mathscr{F}$. The second step is to construct an analytic function $q: V \subset D \rightarrow H^{\infty}$ which contains information concerning the commutant and to show that $q$ is single valued on any domain on which it can be defined. The third step is to show that $q$ can be defined on all of $D$. The final step is to construct $\psi$ in $H^{\infty}(\Omega)$ from $q$ and to show that $\cap_{h \in \mathcal{F}}\left\{T_{h}\right\}^{\prime}=\left\{T_{\psi \circ f}\right\}^{\prime}$.

In several of the arguments we need the following formulation of a theorem of Rudin from his paper $A$ generalization of a theorem of Frostman [8].

RUdin's THEOREM. For $f$ in $H^{\infty}, \operatorname{Inn}(f-f(\alpha))$ is a Blaschke product with distirct zeroes, except for $\alpha$ in a set of capacity zero.

Proof. In [8] Rudin shows that except for $\beta$ in a set of capacity zero,

$$
\lim _{r \rightarrow 1} \int \log \left|f\left(r e^{i \theta}\right)-\beta\right| d \theta=\int \log \left|f\left(e^{i \theta}\right)-\beta\right| d \theta .
$$

If $I$ is the inner factor of $f-\beta$ and the above equality holds, then 


$$
\lim _{r \rightarrow 1} \int \log \left|I\left(r e^{i \theta}\right)\right| d \theta=0 \text {, }
$$

which means $I$ is a Blaschke product [5]. Since a double zero of $f-f(\alpha)$ is a zero of $f^{\prime}$, there are only countably many $\alpha$ for which $\operatorname{Inn}(f-f(\alpha))$ has multiple zeroes. Thus the above formulation follows immediately from the statement in [8].

LEMMA. Let $E$ be a coanalytic function from a domain $U$ in the plane into $H^{2}$. Assume that, for some point $\alpha_{1}$ in $U$, there is a family $\left\{V_{i} \mid i \in \mathcal{G}\right\}$ of neighborhoods of $\alpha_{1}$, and for each $i$ in $g$ there is a bounded function, $H_{i}$, of $V_{i}$ into $H^{\infty}$ such that the following conditions hold:

(i) For each $i, \lim _{\alpha \rightarrow \alpha_{1}} H_{i}(\alpha)=H_{i}\left(\alpha_{1}\right)$ weakly in $H^{2}$.

(ii) $E(\alpha) \perp H_{i}(\alpha) H^{2}$ for all $\alpha$ in $U \cap V_{i}$ and all $i$ in 9 .

(iii) The subspaces $H_{i}\left(\alpha_{1}\right) H^{2}(i \in \mathscr{G})$ span $H^{2}$.

Then $E$ vanishes identically.

Proof of Lemma. $E\left(\alpha_{1}\right) \perp H_{i}\left(\alpha_{1}\right) H^{2}$ for all $i$ in 9 so $E\left(\alpha_{1}\right)=0$. Let

$$
E_{1}(\alpha)=E(\alpha) /\left(\bar{\alpha}-\bar{\alpha}_{1}\right)=\left(E(\alpha)-E\left(\alpha_{1}\right)\right) /\left(\bar{\alpha}-\bar{\alpha}_{1}\right),
$$

for $\alpha \neq \alpha_{1}$ and $E_{1}\left(\alpha_{1}\right)=E^{\prime}\left(\alpha_{1}\right) . E_{1}$ is coanalytic in $U$, and clearly for $\alpha \neq \alpha_{1}$, $E_{1}(\alpha) \perp H_{i}(\alpha) H^{2}$. Since $H_{i}(\alpha) \rightarrow H_{i}\left(\alpha_{1}\right)$ weakly and since they are bounded in $H^{\infty}$, for each $f$ in $H^{2}, H_{i}(\alpha) f \rightarrow H_{i}\left(\alpha_{1}\right) f$ weakly in $H^{2}$. Since $E_{1}$ is coanalytic in $U, E_{1}(\alpha) \rightarrow E_{1}\left(\alpha_{1}\right)$ in norm. It follows immediately that $\left\langle H_{i}(\alpha) f, E_{1}(\alpha)\right\rangle \rightarrow$ $\left\langle H_{i}\left(\alpha_{1}\right) f, E_{1}\left(\alpha_{1}\right)\right\rangle$, that is, that $\left\langle H_{i}\left(\alpha_{1}\right) f, E_{1}\left(\alpha_{1}\right)\right\rangle=0$. Since this is true for all $f$ in $H^{2}$ and all $i$ in 9 , we have $E^{\prime}\left(\alpha_{1}\right)=E_{1}\left(\alpha_{1}\right)=0$.

But we have seen that $E_{1}$ also satisfies the hypotheses of the lemma, which means that $E_{1}^{\prime}\left(\alpha_{1}\right)=0$, that is, that $E^{\prime \prime}\left(\alpha_{1}\right)=0$. It follows by induction that $E^{(n)}\left(\alpha_{1}\right)=0$ for all positive integers $n$, so $E$ is identically zero.

Proof of THEOREM 4. Let $f, \Omega, \mathcal{F}$, and $\alpha_{1}, \ldots, \alpha_{n}$ be as in the hypotheses of the theorem. Let $\mathfrak{A}=\cap_{h \in \mathscr{F}}\left\{T_{h}\right\}^{\prime}$.

Step I. We will construct an analytic map $p$ from a neighborhood of $\alpha_{1}$ into $H^{\infty}$, and for each $h$ in $\mathcal{F}$, we will construct a map $H_{h}$ from a neighborhood of $\alpha_{1}$ into $H^{\infty}$ so that

(i) $H_{h}(\alpha) \rightarrow H_{h}\left(\alpha_{1}\right)$ weakly as $\alpha \rightarrow \alpha_{1}$, for each $h$ in $\mathscr{F}$,

(ii) $T_{p(\alpha)}^{*} S^{*} K_{\alpha} \perp H_{h}(\alpha) H^{2}$ for each $h$ in $\mathscr{F}$, and each $S$ in $\mathfrak{A}$, and

(iii) the subspaces $H_{h}\left(\alpha_{1}\right) H^{2}(h$ in $\mathscr{F})$ span $H^{2}$.

For each $S$ in $\mathfrak{A}$, we will apply the lemma to the function $T_{p(\alpha)}^{*} S^{*} K_{\alpha}$ to conclude that $S^{*} K_{\alpha} \perp p(\alpha) H^{2}$ for $\alpha$ in some neighborhood of $\alpha_{1}$.

If $\beta$ is a point of the plane, an open simply connected set $W$ will be called a slit neighborhood of $\beta$ if there is an analytic arc $\gamma$ in $\mathbf{C} \backslash W$ with one end at $\beta$ such that $W \cup \gamma$ is a neighborhood of $\beta$. An open set $W$ is called a punctured neighborhood of $\beta$ if $\beta$ is not in $W$ but $W \cup\{\beta\}$ is a neighborhood of $\beta$. Thus 
$D \backslash[0,1)$ and $D \backslash\{0\}$ are, respectively, slit and punctured neighborhoods of 0.

For each $h$ in $\mathscr{F}, h=g_{h} \circ f$, let $\beta_{1}^{h}, \beta_{2}^{h}, \beta_{3}^{h}, \ldots$ be the points of $g_{h}^{-1}\left(h\left(\alpha_{1}\right)\right)$ with each point listed with appropriate multiplicity. We assume that $\beta_{j}^{h}=$ $f\left(\alpha_{j}\right)$ for $j=1,2, \ldots, n$. If $\beta$ is a point of $g_{h}^{-1}\left(h\left(\alpha_{1}\right)\right)$ which occurs with multiplicity $r$, then there is a neighborhood of $\beta$ which $g_{h}$ maps $r$-to-1 onto a neighborhood of $h\left(\alpha_{1}\right)$. We obtain $r$ distinct branches of $g_{h}^{-1}$, single valued in a slit neighborhood of $h\left(\alpha_{1}\right)$, arbitrarily continuable in a punctured neighborhood of $h\left(\alpha_{1}\right)$, and which map this neighborhood into a punctured neighborhood of $\beta$. Therefore corresponding to the points of $g_{h}^{-1}\left(h\left(\alpha_{1}\right)\right)$ there are distinct functions $\eta_{1}^{h}, \eta_{2}^{h}, \eta_{3}^{h}, \ldots$ such that for each $j$ we have

(i) $\eta_{j}^{h}$ is a branch of $g_{h}^{-1} \circ g_{h}$,

(ii) $\eta_{j}^{h}$ is single valued in a slit neighborhood of $f\left(\alpha_{1}\right)$ and arbitrarily continuable in a punctured neighborhood of $f\left(\alpha_{1}\right)$,

(iii) $\eta_{j}^{h}$ and its continuations map this punctured neighborhood onto a punctured neighborhood of $\beta_{j}^{h}$, and

(iv) $\lim _{w \rightarrow f\left(\alpha_{1}\right)} \eta_{j}^{h}(w)=\beta_{j}^{h}$.

We take the neighborhoods sufficiently small so that any continuation of $\eta_{j}^{h}$ in the punctured neighborhood of $f\left(\alpha_{1}\right)$ is one of the functions $\eta_{j^{\prime}}^{h}$ with $\beta_{j}^{h}=\beta_{j^{\prime}}^{h}$.

After perhaps renumbering, we assume that $\eta_{1}, \ldots, \eta_{k^{\prime}}$ are the common branches of $g_{h}^{-1} \circ g_{h}$ for $h$ in $\mathscr{F}$, that is, we assume that for each $h$ in $\mathscr{F}$, $\eta_{j}=\eta_{j}^{h}$ for $j=1, \ldots, k^{\prime}$, and for $j>k^{\prime}$, there is a function $g$ with $g \circ f$ in $\mathscr{F}$ so that $\eta_{j}^{h}$ is not a branch of $g^{-1} \circ g$. Since the identity function is a common branch of the $g_{h}^{-1} \circ g_{h}$ and since each common branch contributes a factor to the greatest common divisor of $\left\{\operatorname{Inn}\left(h-h\left(\alpha_{1}\right)\right) \mid h \in \mathcal{F}\right\}$, we have $1<k^{\prime}<n$. (It is possible that $k^{\prime}<n$.) Let $W$ be a neighborhood of $f\left(\alpha_{1}\right)$ so that $\eta_{1}, \ldots, \eta_{k^{\prime}}$ are single valued in a slit neighborhood obtained from $W$ and are arbitrarily continuable in $W \backslash\left\{f\left(\alpha_{1}\right)\right\}$.

Let $U$ be a neighborhood of $\alpha_{1}$ so that $f(U) \subset W$, and define $p$ on $U$ by $p(\alpha)=\Pi_{j=1}^{k^{\prime}}\left(f-\eta_{j}(f(\alpha))\right)$. Clearly $p$ is well defined in a slit neighborhood of $\alpha_{1}$ obtained from $U$, but since continuation of $p$ in $U \backslash\left\{\alpha_{1}\right\}$ at most reorders the factors in the product, $p$ is a single valued analytic map of $U$ into $H^{\infty}$.

Given $h$ in $\mathscr{F}, h=g_{h} \circ f$, there are (at most) finitely many branches $\eta_{k^{\prime}+1}^{h}, \ldots, \eta_{m}^{h}$ such that for $k^{\prime}<j \leqslant m, \eta_{j}^{h}=f\left(\alpha_{l}\right)$ for some $l, 1 \leqslant l \leqslant n$. Choose a neighborhood $U_{h}$ of $\alpha_{1}$ so that $U_{h} \subset U$ and so that $\eta_{k^{\prime}+1}^{h} \circ f, \ldots, \eta_{m}^{h} \circ f$ are defined in slit neighborhoods obtained from $U_{h}$. Define $p_{h}$ by $p_{h}(\alpha)=\prod_{j=k^{\prime}+1}^{m}\left(f-\eta_{j}^{h}(f(\alpha))\right)$. Since continuing $p_{h}$ in $U_{h} \backslash\left\{\alpha_{1}\right\}$ at most reorders the factors in the product, $p_{h}$ is defined and single valued in $U_{h}$. We define $H_{h}$ by $H_{h}(\alpha)=\left(p(\alpha) p_{h}(\alpha)\right)^{-1}(h-h(\alpha))$. So $H_{h}$ maps $U_{h}$ analytically into $H^{\infty}$ and $H_{h}(\alpha) \rightarrow H_{h}\left(\alpha_{1}\right)$ weakly $\left(H^{2}\right)$ as $\alpha \rightarrow \alpha_{1}$. Moreover, 
since $p\left(\alpha_{1}\right) p_{h}\left(\alpha_{1}\right)$ vanishes at $\alpha_{1}, \alpha_{2}, \ldots, \alpha_{n}$ for each $h$ in $\mathscr{F}$, the subspaces $H_{h}\left(\alpha_{1}\right) H^{2}(h$ in $\mathscr{F})$ span $H^{2}$.

Given an operator $S$ in $\mathfrak{A}$, define $E$ on $U$ by $E(\alpha)=T_{p(\alpha)}^{*} S^{*} K_{\alpha}$. Since $p$ is analytic on $U$ and $\alpha \rightarrow K_{\alpha}$ is coanalytic, $E$ is coanalytic on $U$. We wish to show that $E(\alpha) \perp H_{h}(\alpha) H^{2}$ for $h$ in $\mathscr{F}$. It is sufficient to show that $S^{*} K_{\alpha} \perp$ $(h-h(\alpha))\left(f-\eta_{j}^{h}(\alpha)\right)^{-1} H^{2}$ for $j=k^{\prime}+1, \ldots, m$. If $h$ is in $\mathscr{F}$, and $j>k^{\prime}$, there is a function $g$ so that $g \circ f$ is in $\mathscr{F}$ and so that $\eta_{j}^{h}$ is not a branch of $g^{-1} \circ g$, which means that $g\left(\eta_{j}^{h}(f(\alpha))\right)=g(f(\alpha))$ for at most a discrete subset of $U_{h}$. By Rudin's Theorem, $\operatorname{Inn}(g \circ f-g(f(\alpha)))$ is a Blaschke product for a dense set of $\alpha$ in $U_{h}$. If $g \circ f-g(f(\alpha))$ and $f-\eta_{j}^{h}(f(\alpha))$ have a common zero for some $\alpha$, then $g(f(\alpha))=g\left(\eta_{j}^{h}(f(\alpha))\right)$. Therefore the greatest common divisor of $\operatorname{Inn}(g \circ f-g(f(\alpha)))$ and $\operatorname{Inn}\left(f-\eta_{j}^{h}(f(\alpha))\right)$ is 1 for a dense set of $\alpha$ in $U_{h}$. For $\alpha$ in this subset,

$$
\begin{aligned}
& \left((h-h(\alpha)) H^{2}\right)^{\perp} \cap\left((g \circ f-g(f(\alpha))) H^{2}\right)^{\perp} \\
& =\left(\frac{h-h(\alpha)}{f-\eta_{j}^{h}(\alpha)} H^{2}\right)^{\perp} \cap\left((g \circ f-g(f(\alpha))) H^{2}\right)^{\perp} .
\end{aligned}
$$

The fundamental lemma implies that $S^{*} K_{\alpha}$ is in

$$
\left(\frac{h-h(\alpha)}{f-\eta_{j}^{h}(f(\alpha))} H^{2}\right)^{\perp} \cap\left((g \circ f-g(f(\alpha))) H^{2}\right)^{\perp},
$$

hence that $S^{*} K_{\alpha} \perp(h-h(\alpha))\left(f-\eta_{j}^{h}(f(\alpha))\right)^{-1} H^{2}$, for $\alpha$ in this set. By continuity, this holds for all $\alpha$ in $U_{h}$.

We have verified the hypotheses of the lemma so we conclude that $E$ is identically 0 , that is, that $S^{*} K_{\alpha} \perp p(\alpha) H^{2}$ for all $\alpha$ in $U$.

Step II. Let $U^{\prime}$ be an open subset $U$ such that $\eta_{1}, \ldots, \eta_{k^{\prime}}$ are single valued on $f\left(U^{\prime}\right)$. For $\alpha$ in $U^{\prime}$ and $1 \leqslant l \leqslant k^{\prime}$, let $p_{l}(\alpha)=\Pi_{j \neq l}\left(f-\eta_{j}(f(\alpha))\right)$. We will say $\eta_{l}$ is representing for $\mathfrak{A}$ if for some $S$ in $\mathfrak{A}, T_{p_{I}(\alpha)}^{*} S^{*} K_{\alpha}$ is not identically zero for $\alpha$ in $U^{\prime}$. (If $\eta_{l}$ is representing for $\mathfrak{A}$, by coanalyticity $T_{p_{l}(\alpha)}^{*} S^{*} K_{\alpha}$ is 0 on a discrete set of $\alpha$.) Since $\mathfrak{A} T_{z} \subset \mathfrak{A}$, this is easily seen to be equivalent to the condition that $\mathfrak{A}^{*} K_{\alpha}$ not be orthogonal to $(h-h(\alpha)) /\left(f-\eta_{l}(f(\alpha))\right)$ for some $\alpha$ in $U^{\prime}$ and some $h$ in $\mathscr{F}$. Possibly renumbering, we assume that $\eta_{1}, \ldots, \eta_{k}$ are representing and $\eta_{k+1}, \ldots, \eta_{k^{\prime}}$ are not, and we define $q(\alpha)=$ $\Pi_{j=1}^{k}\left(f-\eta_{j}(f(\alpha))\right)$. The choice of $\eta_{1}, \ldots, \eta_{k}$ means that $T_{q(\alpha)}^{*} S^{*} K_{\alpha}=0$ for $\alpha$ in $U^{\prime}$ and $S$ in $\mathscr{A}$, and that this is not true for polynomials in $f$ which properly divide $q$.

Now $q$ is single valued in any subset of $D$ to which it can be continued. To see this, observe that if $a, b$ are complex numbers, $a \neq b$, then $f-a$ and $f-b$ have no common nonconstant inner divisors, for if $\phi$ divides $f-a$ and $f-b$, then $\phi$ divides their difference, $a-b$. For each $\alpha$ in $D$, since $\mathfrak{A} T_{z} \subset \mathfrak{A}$, 
we see that $\mathfrak{U}^{*} K_{\alpha}$ is invariant under $T_{z}^{*}$, so $\overline{\mathfrak{A}^{*} K_{\alpha}}=\left(\phi H^{2}\right)^{\perp}$ for some inner function, $\phi$. Suppose $q$ can be continued to $\tilde{q}$, each defined and single valued on an open set $\tilde{U}$, and for $\alpha$ in $\tilde{U}, q(\alpha)=\Pi_{j=1}^{k}\left(f-w_{j}(\alpha)\right)$ where $w_{1}(\alpha), \ldots, w_{k}(\alpha)$ are distinct, and $\tilde{q}(\alpha)=\prod_{j=1}^{k}\left(f-\tilde{w}_{j}(\alpha)\right)$ where $\tilde{w}_{1}(\alpha), \ldots, \tilde{w}_{k}(\alpha)$ are distinct. We have $\mathfrak{A}^{*} K_{\alpha} \perp q(\alpha) H^{2}$ and $\mathfrak{A}^{*} K_{\alpha} \perp \tilde{q}(\alpha) H_{2}$, so for each $\alpha$ in $\tilde{U}, q(\alpha)$ and $\tilde{q}(\alpha)$ have a common inner divisor $\phi^{\alpha}$. We can write $\phi^{\alpha}$ uniquely as $\phi^{\alpha}=\phi_{1}^{\alpha} \phi_{2}^{\alpha} \cdots \phi_{k}^{\alpha}$ where $\phi_{j}^{\alpha}$ divides $f-w_{j}(\alpha)$. Since each of $\eta_{1}, \ldots, \eta_{k}$ is representing, except for $\alpha$ in a discrete subset of $\tilde{U}$, none of $\phi_{j}^{\alpha}$ is constant. Given $j, 1 \leqslant j \leqslant k$, since $\phi_{j}^{\alpha}$ divides $\tilde{q}(\alpha)$, there is a $j^{\prime}$ so that $\phi_{j}^{\alpha}$ and $f-\tilde{w}_{j^{\prime}}(\alpha)$ have a nonconstant common factor. Thus $f-w_{j}(\alpha)$ and $f-\tilde{w}_{j^{\prime}}(\alpha)$ have a nonconstant common factor and $w_{j}(\alpha)=\tilde{w}_{j^{\prime}}(\alpha)$. Since $\left\{w_{1}(\alpha), \ldots, w_{k}(\alpha)\right\}=\left\{\tilde{w}_{1}(\alpha), \ldots, \tilde{w}_{k}(\alpha)\right\}$ for a dense set of $\alpha$ in $\tilde{U}$, we have $q=\tilde{q}$ on $\tilde{U}$. Therefore $q$ is single valued on any set to which it can be continued.

Step III. Let $V$ be a maximal subdomain of $D$ in which $q$ is continuable. We have seen that $q$ is single valued on $V$. We wish to show that $V=D$.

Suppose, on the contrary, that $D \cap \partial V$ is not empty. Since bounded, single valued analytic functions can be continued across sets of capacity zero, the set $D \cap \partial V$ has positive capacity. (The boundedness of $q$ is immediate from the observation that $\eta_{j}(\alpha)$ is in $\bar{\Omega}$ for all $j$ and $\alpha$.)

Choose $h$ in $\mathscr{F}$ and let $h=g \circ f$. We will find $k$ branches of $g^{-1} \circ g$ defined at some points in $f(D \cap \partial V)$ which are continuations of the branches defining $q$. Using these, $q$ can be extended to an open set containing $V$, which contradicts the maximality of $V$.

Let $L_{1}=\{\beta \mid \beta \in D \cap \partial V$ and $\operatorname{Inn}(h-h(\beta))$ is a Blaschke product with distinct zeroes $\}$. By Rudin's Theorem, $(D \cap \partial V) \backslash L_{1}$ has capacity zero, so $L_{1}$ has positive capacity and is therefore uncountable. Choose $\beta_{1}$ in $L_{1}$ so that every neighborhood of $\beta_{1}$ contains an uncountable subset of $L_{1}$. Suppose $\zeta$ is a branch of $g^{-1} \circ g$ and $U_{\zeta}$ is a neighborhood of $\beta_{1}$ so that $\zeta \circ f$ is single valued on $U_{\zeta}$. (Since the zeroes of $h-h\left(\beta_{1}\right)$ are distinct, such a neighborhood exists for any branch of $g^{-1} \circ g$ defined near $f\left(\beta_{1}\right)$.) For $\alpha$ in $D$, let $\phi^{\alpha}$ be an inner function so that $\left(\mathfrak{U}^{*} K_{\alpha}\right)^{\perp}=\phi^{\alpha} H^{2}$. By the fundamental lemma $\phi^{\alpha}$ divides $h-h(\alpha)$ and by the definition of $q$, for $\alpha$ in $V, \phi^{\alpha}$ divides $q(\alpha)$ For any $S$ in $\mathfrak{A}$, the map

$$
\alpha \rightarrow\left\langle\frac{h-h(\alpha)}{f-\zeta(f(\alpha))}, S^{*} K_{\alpha}\right\rangle
$$

is analytic in $U_{\zeta}$. If there is an $S$ in $\mathfrak{A}$ for which this function is not identically zero, then except for a discrete set of $\alpha, \phi^{\alpha}$ divides $h-h(\alpha)$ but does not divide $(h-h(\alpha)) /(f-\zeta(f(\alpha)))$, which means $\phi^{\alpha}$ and $\operatorname{Inn}(f-\zeta(f(\alpha)))$ have a common factor. In this case, since $\phi^{\alpha}$ divides $q(\alpha)$, it follows that, 
except for a discrete subset of $U_{\zeta} \cap V, \operatorname{Inn}(q(\alpha))$ and $\operatorname{Inn}(f-\zeta(f(\alpha)))$ have a nontrivial common factor. Since $q(\alpha)$ is a polynomial in $f$, we have seen in Step II that this means $f-\zeta(f(\alpha))$ divides $q(\alpha)$. Since the degree of $q$ is $k$, there are at most $k$ such branches of $g^{-1} \circ g$, say $\zeta_{1}, \ldots, \zeta_{r_{1}}, 1<r_{1}<k$. For $\alpha$ in $\cap_{j=1}^{r_{1}} U_{\xi_{j}}$ we define $q_{1}(\alpha)=\prod_{j=1}^{r_{1}}\left(f-\zeta_{j}(f(\alpha))\right)$. Since each factor of $q_{1}$ divides $q$ and since the factors are distinct for $\alpha$ near $\beta_{1}$, we see that $q_{1}$ divides $q$. From the definition of $\phi^{\beta_{1}}$ and the fundamental lemma, we see that $\phi^{\beta_{1}}$ divides $h-h\left(\beta_{1}\right)$. Therefore, $\phi^{\beta_{1}}$ is a Blaschke product and if $\alpha_{0}$ is a zero of $\phi^{\beta_{1}}$, there is a branch $\zeta$ of $g^{-1} \circ g$ which maps $f\left(\beta_{1}\right)$ to $f\left(\alpha_{0}\right)$. Since $\operatorname{Inn}\left(f-\zeta\left(f\left(\beta_{1}\right)\right)\right)$ and $\phi^{\beta_{1}}$ have a common factor, $\zeta$ is one of $\zeta_{1}, \ldots, \zeta_{r_{1}}$. Thus $\phi^{\beta_{1}}$ divides $q_{1}\left(\beta_{1}\right)$, which means $T_{q_{1}\left(\beta_{1}\right)^{2}}^{\mathfrak{I} *} K_{\beta_{1}}=0$. Let $L_{2}=\left\{\beta \mid \beta \in L_{1}\right.$ $\cap\left(\cap_{j=1}^{r_{1}} U_{\xi_{j}}\right)$ and $\left.T_{q_{1}(\beta)^{*}}^{* \mathfrak{A}^{*}} K_{\beta} \neq 0\right\}$. Since $L_{1} \cap\left(\cap_{j=1}^{r_{1}} U_{\xi,}\right)$ is uncountable and since $\alpha \rightarrow T_{q_{1}(\alpha)}^{*} S^{*} K_{\alpha}$ is coanalytic for each $S$ in $\mathfrak{A}$, either $L_{2}$ is empty or $L_{2}$ is uncountable. If $L_{2}$ is not empty, choose $\beta_{2}$ in $L_{2}$ so that each neighborhood of $\beta_{2}$ contains an uncountable subset of $L_{2}$. Define $\zeta_{r_{1}+1}, \ldots, \zeta_{r_{2}}$ and $U_{\gamma_{r_{1}+1}}, \ldots, U_{\xi_{r_{2}}}$ as above and let $q_{2}(\alpha)=\Pi_{j=1}^{r_{2}}\left(f-\zeta_{j}(f(\alpha))\right)$ for $\alpha$ in $\bigcap_{j=1}^{r_{2}} U_{\xi_{j}}$. As in the argument above for $\beta_{1}$, we see that $T_{q_{2}}^{*}\left(\beta_{2}\right) \mathfrak{A}^{*} K_{\beta_{2}}=0$. But $\beta_{2}$ is in $L_{2}$, so $T_{q_{1}\left(\beta_{2}\right)}^{*} \mathfrak{A}^{*} K_{\beta_{2}} \neq 0$ which means that the degree of $q_{2}$ is strictly larger than the degree of $q_{1}$ (as polynomials in $f$ ).

Since the degree of $q$ is $k$, after $s$ steps, $s \leqslant k$, we have $L_{s+1}=\varnothing$. That is, we have $q_{s}$ defined on $\cap_{j=1}^{r_{j}} U_{\zeta_{j}}$ so that on $V \cap\left(\cap_{j=1}^{r_{j}} U_{\zeta_{j}}\right), q_{s}$ divides $q$ and for $\alpha$ in $L_{s} \cap\left(\cap_{j=1}^{r_{s}} U_{\xi_{j}}\right)$ (which is uncountable) $T_{q_{s}(\alpha)}^{*} \mathfrak{I}^{*} K_{\alpha}=0$. But this means that $T_{q_{s}(\alpha)}^{*} \mathfrak{2}{ }^{*} K_{\alpha} \equiv 0$ on $\cap_{j=0}^{r_{j}} U_{\xi_{j}}$, and that $q$ divides $q_{s}$ where both are defined. Since $q$ and $q_{s}$ are monic polynomials (in $f$ ) which divide each other, they are equal, so $q_{s}$ is a continuation of $q$. That is, $q$ is continuable to $V \cup\left(\cap r_{j=0}^{r_{j}} U_{\zeta_{j}}\right)$ which contradicts the definition of $V$. Thus $V=D$.

Step IV. In order to show that the function $\psi$ defined below has the required properties, we must show that the branches $\eta_{1}, \ldots, \eta_{k}$ are a "group" under composition. More precisely, we show that if $\eta_{1}, \ldots, \eta_{k}$ are representing and single valued in $\tilde{U}$ and if $\tilde{\eta}$ is a continuation of one of them to $\eta_{j}(\tilde{U})$, then $\tilde{\eta} \circ \eta_{j}$ is one of the $\eta_{1}, \ldots, \eta_{k}$. Since there are only finitely many branches, one of which is the identity, it follows that for each representing branch $\eta_{j}$, there is a continuation, $\tilde{\eta}$, of one of the $\eta_{1}, \ldots, \eta_{k}$ so that $\tilde{\eta} \circ \eta_{j}(\alpha)=\alpha$ for $\alpha$ in $\tilde{U}$. (Indeed, continuing all the branches along the same path to $\eta_{j}(\tilde{U})$, we obtain $k$ distinct branches on $\eta_{j}(\tilde{U})$, hence $k$ distinct compositions with $\eta_{j}$. Since $\eta_{1}, \ldots, \eta_{k}$ are the only representing branches in $\tilde{U}$, each of these is one of the compositions.)

Choose $h$ in $\mathscr{F}$. Since $\eta_{j}$ is representing, there is an operator $S$ in $\mathfrak{A}$ for which the map

$$
\alpha \rightarrow\left\langle\frac{h-h(\alpha)}{f-\eta_{j}(f(\alpha))}, S^{*} K_{\alpha}\right\rangle
$$


is not identically zero in $U$. By Rudin's Theorem there is an $\tilde{\alpha}$ in $\tilde{U}$ so that $\operatorname{Inn}\left(f-\eta_{j}(f(\tilde{\alpha}))\right)$ is a Blaschke product with distinct zeroes, and so that

$$
T_{(h-h(\tilde{\alpha})) /\left(f-\eta_{y}(f(\tilde{\alpha}))\right)}^{*} S^{*} K_{\tilde{\alpha}} \neq 0 .
$$

Since $\left(f-\eta_{j}(f(\tilde{\alpha}))\right) H^{2 \perp}$ is spanned by $\left\{K_{\beta} \mid f(\beta)=\eta_{j}(f(\tilde{\alpha}))\right\}$, and since $T_{(h-h(\tilde{\alpha})) /(z-\beta)}^{*} S^{*} K_{\beta}$ is a multiple of $K_{\beta}$ whenever $h(\beta)=h(\tilde{\alpha})$, there is $\tilde{\beta}$ so that

$$
T_{(h-h(\tilde{\alpha})) /(z-\tilde{\beta})}^{*} S^{*} K_{\tilde{\beta}}=\tilde{d} K_{\tilde{\beta}} \neq 0 .
$$

We let $\beta(\alpha)$ be the branch of $h^{-1} \circ h$ defined near $\tilde{\alpha}$ so that $\beta(\tilde{\alpha})=\beta$. In a neighborhood of $\tilde{\alpha}$ we get

$$
T_{(h-h(\alpha)) /(z-\beta(\alpha))}^{*} S^{*} K_{\alpha}=d(\alpha) K_{\beta(\alpha)} \quad \text { where } d(\alpha) \neq 0 .
$$

Since $\tilde{\eta}$ is representing in $\eta_{j}(\tilde{U})$ we can find $\tilde{S}$ so that

$$
\alpha \rightarrow\left\langle\frac{h-h(\beta(\alpha))}{f-\tilde{\eta}(f(\beta(\alpha)))}, \tilde{S}^{*} K_{\beta(\alpha)}\right\rangle
$$

is not identically zero. Since $\tilde{\eta}(f(\beta(\alpha)))=\tilde{\eta} \circ \eta_{j}(f(\alpha))$, this means that the map

$$
\left[\alpha \rightarrow\left\langle\frac{h-h(\alpha)}{f-\tilde{\eta} \circ \eta_{j}(f(\alpha))}, \tilde{S}^{*} T_{(h-h(\alpha)) /(z-\beta(\alpha))}^{*} S^{*} K_{\alpha}\right\rangle\right]
$$

is not identically zero, which implies $\tilde{\eta} \circ \eta_{j}$ is representing on a subset of $U$, and is therefore one of $\eta_{1}, \ldots, \eta_{k}$.

Choose a point $w_{0}$ of $\Omega$ so that $\eta_{1}\left(w_{0}\right), \ldots, \eta_{k}\left(w_{0}\right)$ are distinct. We define $\psi$ by $\psi(w)=\Pi_{j=1}^{k}\left(w_{0}-\eta_{j}(w)\right)$, that is, $\psi(f(\alpha))=q(\alpha)\left(z_{0}\right)$ where $f\left(z_{0}\right)=w_{0}$. Since $q$ is single valued on $D$, and $q(\alpha)=q(\beta)$ whenever $f(\alpha)=f(\beta), \psi$ is single valued on $\Omega$. Moreover, the "group" property shows that $\psi \circ \eta_{j}=\psi$ for $j=1, \ldots, k$.

Choose $\delta>0$ so that on the neighborhood $N, N=\left\{w|| w-w_{0} \mid<\delta\right\}, \psi$ is univalent, the $\eta_{j}$ are single valued, univalent, and the $\eta_{j}(N)$ are disjoint.

If $c$ is a complex number with $|c|<\delta^{k}$, we show that $\psi^{-1}(c)$ consists of exactly $k$ points. If $w$ is in $\Omega$ and $\psi(w)=c$, then for at least one branch $\eta$ defined near $w$ we have $\left|\eta(w)-w_{0}\right|<\delta$, so $\eta(w)$ is in $N$. Since $\psi$ is univalent on $N$ and since $\psi \circ \eta_{j}=\psi$, we have $\psi^{-1}(c)=\left\{\eta_{1}(\eta(w))\right.$, $\left.\eta_{2}(\eta(w)), \ldots, \eta_{k}(\eta(w))\right\}$.

Let $\tilde{N}$ be the set of $\alpha$ in $D$ such that $|\psi(f(\alpha))|<\delta^{k}$. From the fundamental lemma, $S$ is in $\left\{T_{\psi \circ f}\right\}^{\prime}$ if and only if for all $\alpha$ in $\tilde{N}$,

$$
S^{*} K_{\alpha} \perp(\psi \circ f-\psi \circ f(\alpha)) H^{2} .
$$

But for each $\alpha$ in $\tilde{N}$, we have from the above description of $\psi^{-1}(\psi(f(\alpha)))$ that the map 


$$
w \rightarrow(\psi(w)-\psi(f(\alpha))) / \prod_{j=1}^{k}\left(w-\eta_{j}(f(\alpha))\right)
$$

is invertible in $H^{\infty}(\Omega)$, which means that for $\alpha$ in $\tilde{N}$,

$$
(\psi \circ f-\psi(f(\alpha))) H^{2 \perp}=\left(\prod_{j=1}^{k}\left(f-\eta_{j}(f(\alpha))\right)\right) H^{2 \perp}=\left(q(\alpha) H^{2}\right)^{\perp} .
$$

Therefore, $S$ is in $\left\{T_{\psi \circ f}\right\}^{\prime}$ if and only if $S^{*} K_{\alpha} \perp q(\alpha) H^{2}$ for $\alpha$ in $\tilde{N}$, which happens if and only if $S$ is in $\mathfrak{A}$.

This theorem is of interest from at least two standpoints. First, it gives sufficient conditions for the intersections of commutants to be the same as the commutant of a single operator. This naturally leads to the question: If $\mathscr{F}$ is any family of functions in $H^{\infty}$, does there exists $\psi$ in $H^{\infty}$ so that $\cap_{h \in \mathcal{F}}\left\{T_{h}\right\}^{\prime}=\left\{T_{\psi}\right\}^{\prime}$ ?

Secondly, even when $\mathcal{F}$ consists of a single function, $g \circ f$, the theorem gives a possibly simpler function $\psi$, so that $\left\{T_{\psi \circ f}\right\}^{\prime}=\left\{T_{g \circ f}\right\}^{\prime}$. It is clear from the proof that $\psi$ is constructed from some of the branches of $g^{-1} \circ g$ at $f\left(\alpha_{1}\right)$ which can be continued (almost everywhere) throughout $D$. The function, constructed similarly, using all such branches behaves similarly (these may be different functions), so we obtain such a $\psi$ explicitly by finding all such branches.

It is also clear from the proof that the number $w_{0}$ is fairly arbitrary, and that different choices give different functions. That it is not completely arbitrary can be seen from the following example. Let $\Omega$ be the annulus $\Omega=\left\{z\left|\frac{1}{2}<\right| z \mid<2\right\}$ and let $g$ be the function $g(z)=z+1 / z$. Then there are two branches of $g^{-1} \circ g$ continuable in $\Omega$, namely, $\eta_{1}(\alpha)=\alpha$ and $\eta_{2}(\alpha)=1 / \alpha$. Taking $w_{0}$ to be 1 , which is allowable in the proof, $\psi(w)=$ $(1-w)(1-1 / w)=2-(w+1 / w)$. However, taking $w_{0}$ to be 0 , which is not allowable, $\psi(w)=(0-w)(0-1 / w) \equiv 1$.

The special case of this theorem when $f(z)=z$ is of particular interest, because in this case the function $\psi$ can be taken to be a finite Blaschke product. Since inner functions are ancestral, it follows immediately that all the functions in $\mathscr{F}$ are composites of $\psi$.

THEOREM 5. Let $\mathcal{F}$ be a family of nonconstant $H^{\infty}$ functions. If for some point $\alpha_{1}$ in $D$, the greatest common divisor of $\left\{\operatorname{Inn}\left(h-h\left(\alpha_{1}\right)\right) \mid h \in \mathcal{F}\right\}$ is a finite Blaschke product, then there is a finite Blaschke product $\psi$ so that $\cap_{h \in \mathfrak{F}}\left\{T_{h}\right\}^{\prime}=\left\{T_{\psi}\right\}^{\prime}$.

Proof. The hypotheses of this theorem are the special case of Theorem 4 when $f$ is the identity function, $f(z)=z$. To prove the stronger conclusion, we need only construct a particular $\psi$. 
In Step IV of the proof of Theorem 4, we wish to let $w_{0}=0$. This is not a loss of generality, for we may choose $h_{0}$ in $\mathscr{F}$ and $\beta$ in $D$ so that $\operatorname{Inn}\left(h_{0}-h_{0}(\beta)\right)$ is a Blaschke product with distinct zeroes (Rudin's Theorem). Let $\mathscr{F}^{\prime}=\{h((z+\beta) /(1+\bar{\beta} z)) \mid h \in \mathscr{F}\}$. Since the zeroes of $h_{0}-$ $h_{0}(\beta)$ are distinct, we may construct $\psi_{1}$ for the family $\mathscr{F}^{\prime}$ using $w_{0}=0$. Then $\psi(w)=\psi_{1}((w-\beta) /(1-\bar{\beta} w))$ solves the problem for $\mathscr{F}$, and $\psi$ is a finite Blaschke product if $\psi_{1}$ is.

Therefore, in Step IV we take $w_{0}=0$, so that $\psi(w)=(-1)^{k} \Pi_{j=1}^{k} \eta_{j}(w)$. We will show that $\psi$ is a finite Blaschke product. Clearly, $\|\psi\|_{\infty}<1$. For $0<r<1$, let

$$
\begin{aligned}
& F_{r}=\{w \mid \text { for some } z,|z| \leqslant r, \text { and some continuation } \eta, \\
& \left.\qquad \text { of } \eta_{1}, \ldots, \eta_{k_{1}}, \eta(z)=w\right\} .
\end{aligned}
$$

That is, $F_{r}$ is the image of the compact set which lies above $\{z|| z \mid \leqslant r\}$ in the Riemann surface for $\left\{\eta_{j}\right\}$, so $F_{r}$ is compact. Let $s(r)=\max \left\{|w| \mid w \in F_{r}\right\}$. It follows from the group-like nature of the $\left\{\eta_{j}\right\}$ proved in Step IV that, if $w$ is in $F_{r}$, so is $\eta^{-1}(w)$, when $\eta$ is any continuation of $\eta_{1}, \ldots, \eta_{k}$. If $|\psi(w)| \leqslant r^{k}$, then for some $\eta,|\eta(w)| \leqslant r$, so $w$ is in $F_{r}$. Thus if $|w|>s(r)$, then $|\psi(w)| \geqslant$ $r^{k}$. That is, $\lim _{\rho \rightarrow 1}\left|\psi\left(\rho \mathrm{e}^{i \theta}\right)\right|=1$ for every $\theta$, and is uniform in $\theta$, so $\psi$ is a finite Blaschke product.

COROLlary. If $h$ is in $H^{\infty}$, and for some $\alpha_{1}$ in $D$, $\operatorname{Inn}\left(h-h\left(\alpha_{1}\right)\right)$ is a finite Blaschke product, then there is a finite Blaschke product $\psi$ so that $\left\{T_{h}\right\}^{\prime}=$ $\left\{T_{\psi}\right\}^{\prime}$.

Proof. In Theorem 5, let $\mathcal{F}=\{h\}$.

This corollary seems to be an improvement of a theorem of Thomson [15]. Thomson proves "If $\operatorname{Inn}(f-f(\alpha))$ is a finite Blaschke product for uncountably many $\alpha$ in $D$, then there is a finite Blaschke product $\phi$ such that $\left\{T_{f}\right\}^{\prime}=\left\{T_{\phi}\right\}^{\prime}$." However, I do not know any function $f$ for which $\{\alpha \mid \operatorname{Inn}(f$ $-f(\alpha)$ ) is a finite Blaschke product $\}$ is a nonempty countable set. If there are no functions with this property, every function which satisfies the hypotheses of the corollary also satisfies the hypotheses of Thomson's theorem.

As a final illustration of these techniques, we give a generalization of a theorem of Thomson [15]. The result uses a symmetry condition. If $k$ is a positive integer, we say $f$ is $k$-symmetric if $\alpha$ in $D$ and $\lambda^{k}=1$ imply $f-\lambda f(\alpha)$ is not an outer function.

COROLLARY. If $f$ is in $H^{\infty}$ and $n$ is a positive integer, then $\left\{T_{f^{n}}\right\}^{\prime}=\left\{T_{f^{m}}\right\}^{\prime}$ where $m$ is the least common multiple of $\{k \mid k$ divides $n$ and $f$ is $k$-symmetric $\}$.

Proof. Let $\lambda$ be a primitive $n$th root of 1 . If $g(w)=w^{n}$, then the branches 
of $g^{-1} \circ g$ are $\eta_{j}(w)=\lambda^{j} w, j=1,2, \ldots, n$. In Step II of the proof of Theorem 4, we saw that $S$ commutes with $T_{f^{n}}$ if and only if for every $\alpha$ in $D$, $S^{*} K_{\alpha} \perp q(\alpha) H^{2}$ where $q$ was defined by $q(\alpha)=\Pi_{l=1}^{r}\left(f-\eta_{j}(f(\alpha))\right)$ where $\eta_{j i}$, $\eta_{j_{2}}, \ldots, \eta_{j_{1}}$ are representing branches of $g^{-1} \circ g$. Since $m$ divides $n$, we have $\left\{T_{f^{n}}\right\}^{\prime} \supset\left\{T_{f^{m}}\right\}^{\prime}$. Thus, it is sufficient to show that $q(\alpha)$ divides $f^{m}-f(\alpha)^{m}$, that is, that the $n$th roots of 1 corresponding to representing branches are actually $m$ th roots of 1 .

Suppose $\eta_{l}$ is representing and $\lambda^{\prime}$ is a primitive $k$ th root of 1 . By the group property proved in Step IV, $\eta_{l} \circ \eta_{l}=\eta_{2 l}, \eta_{l} \circ \eta_{l} \circ \eta_{l}=\eta_{3 l}, \ldots$ are all representing. If $f$ is not $k$-symmetric, then there exist $\mu, \mu=\lambda^{l s}$, and $\alpha_{1}$ in $D$ so that $f-\mu f\left(\alpha_{1}\right)$ is outer. But the proof of Step I shows that in this case $\eta_{s l}$ cannot be representing, which contradicts the assumption that $\eta_{l}$ is representing. Thus $f$ is $k$-symmetric, and $\lambda^{\prime}$ is an $m$ th root of 1 .

Thomson's theorem [15] is: "If $f$ is univalent and nonvanishing, then $\left\{T_{f^{n}}\right\}^{\prime}=\left\{T_{f}\right\}^{\prime}$. The above corollary includes Thomson's result because a univalent nonvanishing function is $k$-symmetric if and only if $k=1$. Easy examples show that the integer $m$ of the corollary may not be the least integer $k$ such that $\left\{T_{f^{n}}\right\}^{\prime}=\left\{T_{f^{k}}\right\}^{\prime}$, but this is probably the best result based only on symmetry conditions.

4. In this section we will describe the commutant of an analytic Toeplitz operator whose symbol is a covering map. In particular, we will show that covering maps are ancestral, so the results of $\S 1$ show that these functions give rise to a new class of distinct commutants. Abrahamse [1] and Abrahamse and Ball [2] have used this kind of example to answer two questions of Deddens and Wong [4]. The techniques of the previous sections suggest covering maps as appropriate objects of study since the branches of $\phi^{-1} \circ \phi$ are particularly simple when $\phi$ is a covering map.

Suppose $\Omega$ is a bounded domain in the plane. Then $\Omega$ is a Riemann surface and its universal covering space is the disk. The Koebe Uniformization Theorem says that given $\alpha_{0}$ in $D$ and $w_{0}$ in $\Omega$, there is a unique covering map $\phi$ of $D$ onto $\Omega$ with $\phi\left(\alpha_{0}\right)=w_{0}$ and $\phi^{\prime}\left(\alpha_{0}\right)>0$. Because $\phi$ is a covering, $\phi^{-1}$ is arbitrarily continuable in $\Omega$, so each branch of $\phi^{-1} \circ \phi$ is arbitrarily continuable in $D$. Since $D$ is simply connected, each branch of $\phi^{-1} \circ \phi$ is a single valued map of $D$ into $D$. It follows from the Koebe Uniformization Theorem that the set of branches of $\phi^{-1} \circ \phi$ is the set of linear fractional transformations, $I$, which map $D$ onto $D$, and have the property $\phi \circ I=\phi$. This set of linear fractional transformations is a group under composition, indeed it is the group of deck transformations of $D$ regarded as the universal covering space of $\Omega$, and is therefore isomorphic to the fundamental group of $\Omega$. For example, if $\Omega$ is the annulus $\Omega=\left\{z\left|\frac{1}{2}<\right| z \mid<2\right\}$, a covering map $\phi$ is the composition of a univalent map of $D$ onto the strip $\{z \mid-\ln 2<\operatorname{Re} z<$ 
$\ln 2$ \} and the exponential map. The group of linear fractional transformations of $D$ corresponds (under the univalent map) to the group of translations in the strip by $2 \pi k i, k$ an integer. More information on covering transformations and a proof of the Koebe Uniformization Theorem can be found in standard references on complex analysis or Riemann surfaces, for example Veech's $\boldsymbol{A}$ second course in complex analysis [16].

Throughout this section, $\Omega$ will be a bounded domain in the plane, $\phi$ will be a covering map of $D$ onto $\Omega$, and $G$ will be the group of linear fractional transformations, $I$, which map $D$ onto $D$ and satisfy $\phi \circ I=\phi$. The case of a simply connected domain, $\Omega$, is trivial from our point of view, since in this case $\phi$ is univalent, $G$ consists only of the identity, and $\left\{T_{\phi}\right\}^{\prime}=\left\{T_{z}\right\}^{\prime}$. This case will be tacitly excluded.

The following theorem provides the foundation for the proof of Theorem 7, but it is also of interest from a function theoretic point of view. (T. A. Metzger has pointed out that this theorem was discovered by E. L. Stout [17].)

THEOREM 6. Let $\phi$ be a covering map from $D$ onto the bounded domain $\Omega$. For each $\alpha$ in $D, \operatorname{Inn}(\phi-\phi(\alpha))$ is an interpolating Blaschke product.

PRoof. Let $\alpha_{0}$ be in $D$. Without loss of generality, we may assume $\phi\left(\alpha_{0}\right)=0$. For $r>0$, let $D_{r}=\{z|| z \mid<r\}$. Choose $\delta$ and $\delta_{1}$ so that $0<\delta<$ $\delta_{1}$ and $D_{\delta_{1}} \subset \Omega$. Since $D_{\delta_{1}}$ is simply connected and each branch of $\phi^{-1}$ is arbitrarily continuable in $D_{\delta_{1}}$, each branch of $\phi^{-1}$ is single valued in $D_{\delta_{1}}$, so $D_{\delta_{1}}$ is evenly covered by $\phi$. That is, $\phi^{-1}\left(D_{\delta_{1}}\right)=\cup_{n=0}^{\infty} U_{n}$ where the $U_{n}$ are disjoint open sets and $\phi \mid U_{n}$ is a univalent map of $U_{n}$ onto $D_{\delta_{1}}$. Let $\left\{\alpha_{n}\right\}_{n=0}^{\infty}=$ $\phi^{-1}\left(\phi\left(\alpha_{0}\right)\right)$ where $\alpha_{n}$ is in $U_{n}$ for each $n$, and let $\psi$ be a Blaschke product whose zero sequence is $\left\{\alpha_{n}\right\}_{n=0}^{\infty}$. Obviously $\psi$ divides $\phi$. We will show that $\phi / \psi$ is invertible in $H^{\infty}$, hence outer, so that $\psi$ is the inner factor of $\phi$, and also that $\left\{\alpha_{n}\right\}_{n=0}^{\infty}$ is an interpolating sequence.

Let $V_{n}=U_{n} \cap \phi^{-1}\left(D_{\delta}\right)$. If $z$ is in $D \backslash \cup_{n=0}^{\infty} V_{n}$, then $|\phi(z) / \psi(z)|>|\phi(z)|$ $>\delta$ since $\phi(z)$ is not in $D_{\delta}$. Now $\phi(z) / \psi(z)$ is continuous on $\bar{V}_{0}$ and since $\alpha_{0}$ is the only zero of $\phi$ in $\bar{V}_{0}$ and $\phi\left(\alpha_{0}\right) / \psi\left(\alpha_{0}\right) \neq 0$, it follows that $\phi(z) / \psi(z) \neq 0$ for $z$ in $\bar{V}_{0}$. Thus $\varepsilon=\min _{z \in \bar{V}_{0}}|\phi(z) / \psi(z)|$ is greater than zero.

If $I$ is the linear fractional transformation which maps $\alpha_{k}$ to $\alpha_{0}$ and has the property $\phi \circ I=\phi$, then $I$ maps $\bar{V}_{k}$ onto $\bar{V}_{0}$. Since $\psi$ is a Blaschke product with zeroes $\left\{\alpha_{n}\right\}_{n=0}^{\infty}, \psi \circ I$ is a Blaschke product with zeroes $\left\{I^{-1}\left(\alpha_{n}\right)\right\}_{n=0}^{\infty}=$ $\left\{\alpha_{n}\right\}_{n=0}^{\infty}$, so $|\psi(z)|=|\psi \circ I(z)|$ for all $z$ in $D$. If $z$ is in $\bar{V}_{k}$, then $I(z)$ is in $\bar{V}_{0}$ and

$$
\left|\frac{\phi(z)}{\psi(z)}\right|=\left|\frac{\phi(I(z))}{\psi(I(z))}\right|>\varepsilon .
$$

Thus if $z$ is in $\cup_{n=0}^{\infty} V_{n},|\phi(z) / \psi(z)|>\varepsilon$, so we have 


$$
\left|\frac{\phi(z)}{\psi(z)}\right| \geqslant \min (\delta, \varepsilon) \text { for all } z \text { in } D,
$$

that is, $\phi / \psi$ is invertible in $H^{\infty}$.

To see that $\left\{\alpha_{n}\right\}_{n=0}^{\infty}$ is an interpolating sequence, it is sufficient to show that $\inf _{n}\left|\psi^{\prime}\left(\alpha_{n}\right)\right|\left(1-\left|\alpha_{n}\right|^{2}\right)$ is nonzero. (See [5, pp. 194ff].) We have seen that $\psi(z)=\lambda \psi(I(z))$ for some $\lambda,|\lambda|=1$. Therefore

$$
\psi^{\prime}\left(\alpha_{k}\right)=\lambda \psi^{\prime}\left(I\left(\alpha_{k}\right)\right) I^{\prime}\left(\alpha_{k}\right)=\lambda \psi^{\prime}\left(\alpha_{0}\right) I^{\prime}\left(\alpha_{k}\right)
$$

Since $I$ is a linear fractional transformation which maps $D$ onto $D$ and $I\left(\alpha_{k}\right)=\alpha_{0}$, a calculation shows $\left|I^{\prime}\left(\alpha_{k}\right)\right|=\left(1-\left|\alpha_{0}\right|^{2}\right) /\left(1-\left|\alpha_{k}\right|^{2}\right)$. Thus

$$
\left|\psi^{\prime}\left(\alpha_{k}\right)\right|\left(1-\left|\alpha_{k}\right|^{2}\right)=\left|\psi^{\prime}\left(\alpha_{0}\right)\right|\left(1-\left|\alpha_{0}\right|^{2}\right) \text {, }
$$

which means

$$
\inf _{n}\left|\psi^{\prime}\left(\alpha_{n}\right)\right|\left(1-\left|\alpha_{n}\right|^{2}\right)=\left|\psi^{\prime}\left(\alpha_{0}\right)\right|\left(1-\left|\alpha_{0}\right|^{2}\right)>0
$$

COROLlARY. If $\phi$ is a covering map of $D$ onto $\Omega$, then $\phi$ is ancestral.

Proof. For $I$ in $G$ define the operator $S_{I}$ on $H^{2}$ by $S_{I}(h)=h \circ I$. Ryff [9] and Nordgren [6] have shown that $S_{I}$ is a bounded operator, and since $\phi \circ I=\phi$, we see that $S_{I}$ commutes with $T_{\phi}$. An easy calculation shows that $S_{I}^{*} K_{\alpha}=K_{I(\alpha)}$. So the closed span of $\left\{S_{I}^{*} K_{\alpha}\right\}$ for $I$ in $G$ is $(\phi-\phi(\alpha)) H^{2 \perp}$, and $\phi$ is ancestral.

Therefore, the results of $\S \S 1$ and 5 concerning ancestral functions apply to covering maps. For the most part, but not always, covering maps give rise to commutants distinct from those from inner functions. Indeed, if $\phi$ is a covering of $D$ onto $\Omega$, there is an inner function $\psi$ such that $\left\{T_{\psi}\right\}^{\prime}=\left\{T_{\phi}\right\}^{\prime}$ if and only if there is a simply connected domain $\tilde{\Omega}$ containing $\Omega$ such that $\tilde{\Omega} \backslash \Omega$ has capacity zero. Given such a domain $\tilde{\Omega}$, if $h$ is a univalent map of $\tilde{\Omega}$ onto $D$, then $\psi=h \circ \phi$ is an inner function and $\left\{T_{\psi}\right\}^{\prime}=\left\{T_{\phi}\right\}^{\prime}$. Conversely, suppose $\psi$ is an inner function and $\phi$ is a covering of $D$ onto $\Omega$ with $\left\{T_{\psi}\right\}^{\prime}=\left\{T_{\phi}\right\}^{\prime}$. Since $\phi$ and $\psi$ are ancestral, there is a univalent function $h$ which maps $\Omega$ onto $\psi(D)$ so that $\psi=h \circ \phi$. Since $\psi$ is inner, $D \backslash \psi(D)$ has capacity zero and $h^{-1}$ can be extended to all of $D$. If we let $\tilde{\Omega}=h^{-1}(D)$, then $\tilde{\Omega}$ is simply connected, contains $\Omega$ and $\tilde{\Omega} \backslash \Omega$ has capacity zero. Abrahamse [1] was the first to show that not every commutant of an analytic Toeplitz operator is the same as the commutant of a Toeplitz operator whose symbol is inner, which answered a question of Deddens and Wong [4]. His example was a covering map of the disk onto an annulus. The above result shows that this example is not extraordinary but is one of a large family of distinct commutants.

Theorem 6 is most significant for us because if $\left\{\alpha_{n}\right\}_{n=0}^{\infty}$ is an interpolating 
sequence and $\psi$ is a Blaschke product with zero set $\left\{\alpha_{n}\right\}_{n=0}^{\infty}$, then the functions $\sqrt{1-\left|\alpha_{n}\right|^{2}} K_{\alpha_{n}}$ are a basis, equivalent in the Banach space sense to an orthonormal basis, for the subspace $H^{2} \Theta \psi H^{2}$. This result follows immediately from a theorem of Shapiro and Shields [10]. Letting $\left\{e_{n}\right\}_{n=0}^{\infty}$ denote the usual orthonormal basis for $l^{2}$, Shapiro and Shields show that the map $\Phi: H^{2} \Theta \psi H^{2} \rightarrow l^{2}$ given by $\Phi f=\sum_{n=0}^{\infty} \sqrt{1-\left|\alpha_{n}\right|^{2}} f\left(\alpha_{n}\right) e_{n}$ is an isomorphism. Therefore $\Phi^{*}: l^{2} \rightarrow H^{2} \ominus \psi H^{2}$ is an isomorphism, and a simple calculation shows $\Phi^{*} e_{n}=\sqrt{1-\left|\alpha_{n}\right|^{2}} K_{\alpha_{n}}$. In particular, any function, $h$, in $H^{2} \Theta \psi H^{2}$ has a unique expression of the form $h=\sum_{n=0}^{\infty} d_{n} K_{\alpha_{n}}$ where the series converges in norm.

THEOREM 7. Suppose $\Omega$ is a bounded domain in the plane, $\phi$ is a covering map of $D$ onto $\Omega$, and $G$ is the group of linear fractional transformations, $I$, such that $\phi \circ I=\phi$.

If $S$ is in $\left\{T_{\phi}\right\}^{\prime}$ then for each $I$ in $G$ there is an analytic function, $C_{I}$, defined on $D$ so that for each $\alpha$ in $D$ and each $g$ in $H^{2}$,

$$
(S g)(\alpha)=\sum_{I \in G} C_{I}(\alpha) g(I(\alpha))
$$

where the series converges absolutely for each $\alpha$ and uniformly on compact subsets of $D$.

Conversely, if $S$ is a bounded operator on $H^{2}$ which has a representation (*), then $S$ commutes with $T_{\phi}$.

Proof. Suppose $S$ is in $\left\{T_{\phi}\right\}^{\prime}$. By the Fundamental Lemma, for each $\alpha$ in $D, S^{*} K_{\alpha}$ is orthogonal to $(\phi-\phi(\alpha)) H^{2}$. Theorem 6 and the remark above show that for each $\alpha$ in $D$, there are scalars, $C_{I}(\alpha)$, so that $S^{*} K_{\alpha}=\Sigma_{I \in G} \overline{C_{I}(\alpha)} K_{I(\alpha)}$ where the series converges in norm. If $g$ is in $H^{2}$, we have

$$
\begin{aligned}
(S g)(\alpha) & =\left\langle S g, K_{\alpha}\right\rangle=\left\langle g, S^{*} K_{\alpha}\right\rangle=\sum_{I \in G} C_{I}(\alpha)\left\langle g, K_{I(\alpha)}\right\rangle \\
& =\sum_{I \in G} C_{I}(\alpha) g(I(\alpha)) .
\end{aligned}
$$

For each $I$ in $G$,

$$
S\left(\frac{\phi-\phi(\alpha)}{z-I(\alpha)}\right)(\alpha)=C_{I}(\alpha) \phi^{\prime}(I(\alpha))
$$

Since $\phi^{\prime}$ does not vanish in $D$, the functions $\alpha \rightarrow C_{l}(\alpha)$ are analytic in $D$.

Conversely, if $S$ is a bounded operator on $H^{2}$ and has a representation (*), then for every $\alpha$ in $D$ and every $g$ in $H^{2}$, 


$$
\begin{aligned}
\left(S T_{\phi} g\right)(\alpha) & =\sum_{I \in G} C_{I}(\alpha) \phi(I(\alpha)) g(I(\alpha)) \\
& =\phi(\alpha) \sum_{I \in G} C_{I}(\alpha) g(I(\alpha))=\left(T_{\phi} S g\right)(\alpha) .
\end{aligned}
$$

Therefore $S T_{\phi}=T_{\phi} S$.

It is, of course, of interest to give necessary and sufficient conditions on the functions $C_{I}$ so that the corresponding operator is bounded. Although I have not had notable success along this line, the following theorem gives a weak condition.

THEOREM 8. If $J_{1}, J_{2}, \ldots, J_{N}$ are distinct finite Blaschke products, and $C_{1}, \ldots, C_{N}$ are analytic functions on $D$ so that the operator $S$ defined by

$$
(S g)(\alpha)=\sum_{k=1}^{N} C_{k}(\alpha) g\left(J_{k}(\alpha)\right)
$$

is bounded on $H^{2}$, then each $C_{k}$ is in $H^{\infty}$.

Proof. Clearly

$$
S^{*} K_{\alpha}=\sum_{k=0}^{N} \overline{C_{k}(\alpha)} K_{J_{k}(\alpha)} .
$$

We define $\psi(z)=\prod_{k=1}^{N} J_{k}(z)$ and for each $\alpha$ in $D$ we let

$$
\Phi_{\alpha}(z)=\prod_{k=1}^{N} \frac{z-J_{k}(\alpha)}{1-\overline{J_{k}(\alpha) z}} .
$$

For each $\alpha$ in $D, S^{*} K_{\alpha} \perp \Phi_{\alpha} H^{2}$ and $\left|\Phi_{\alpha}(0)\right|=|\psi(\alpha)|$, so it follows from the lemma of $\$ 2$ that if $f$ is in $H^{\infty}$, then

$$
|(S f)(\alpha)| \leqslant\|S\|\|f\|_{\infty} \sqrt{\frac{1-\left|\Phi_{\alpha}(0)\right|^{2}}{1-|\alpha|^{2}}}=\|S\|\|f\|_{\infty} \sqrt{\frac{1-|\psi(\alpha)|^{2}}{1-|\alpha|^{2}}} .
$$

Since $\psi$ is a finite Blaschke product, $\psi^{\prime}$ is in $H^{\infty}$, and

$$
\begin{aligned}
\lim _{r \rightarrow 1}\left|(S f)\left(r e^{i \theta}\right)\right| & <\lim _{r \rightarrow 1}\|S\|\|f\|_{\infty} \sqrt{\frac{1-\left|\psi\left(r e^{i \theta}\right)\right|^{2}}{1-\left|r e^{i \theta}\right|^{2}}} \\
& <\|S\|\|f\|_{\infty} \lim _{r \rightarrow 1} \sqrt{\frac{\psi\left(e^{i \theta}\right)-\psi\left(r e^{i \theta}\right)}{e^{i \theta}-r e^{i \theta}} \mid} \\
& =\|S\|\|f\|_{\infty} \sqrt{\left|\psi^{\prime}\left(e^{i \theta}\right)\right|}<\|S\|\|f\|_{\infty} \sqrt{\left\|\psi^{\prime}\right\|_{\infty}} .
\end{aligned}
$$

Fix an integer $n, 1<n<N$. For each $\beta$ in $D$ we define 


$$
f_{\beta}(z)=\prod_{\substack{k=1 \\ k \neq n}}^{N} \frac{z-J_{k}(\beta)}{1-\overline{J_{k}(\beta)} z} .
$$

So $f_{\beta}$ is a finite Blaschke product, and $\left\|f_{\beta}\right\|_{\infty}=1$. The above calculation shows that $\left|\left(S f_{\beta}\right)(\beta)\right|<\|S\| \sqrt{\left\|\psi^{\prime}\right\|_{\infty}}$. On the other hand the definition of the operator $S$ means

$$
\left(S f_{\beta}\right)(\beta)=\sum_{k=1}^{N} C_{k}(\beta) f_{\beta}\left(J_{k}(\beta)\right)=C_{n}(\beta) f_{\beta}\left(J_{n}(\beta)\right) .
$$

It follows that

$$
\left|C_{n}(\beta)\right|<\frac{\|S\| \sqrt{\left\|\psi^{\prime}\right\|_{\infty}}}{\left|f_{\beta}\left(J_{n}(\beta)\right)\right|}=\|S\| \sqrt{\left\|\psi^{\prime}\right\|_{\infty}}\left|\prod_{\substack{k=1 \\ k \neq n}}^{N} \frac{1-J_{n}(\beta) \overline{J_{k}(\beta)}}{J_{k}(\beta)-J_{n}(\beta)}\right| .
$$

Since $J_{1}, \ldots, J_{N}$ are distinct, there are only finitely many points in $\bar{D}$ for which $J_{n}$ is equal to any of the other $J_{k}$, so, except for finitely many radii,

We also have

$$
\limsup _{r \rightarrow 1}\left|C_{n}\left(r e^{i \theta}\right)\right| \leqslant\|S\| \sqrt{\left\|\psi^{\prime}\right\|_{\infty}} \text {. }
$$

$$
\left|C_{n}\left(r e^{i \theta}\right)\right|<2\|S\| \sqrt{\left\|\psi^{\prime}\right\|_{\infty}} \prod_{\substack{k=1 \\ k \neq n}}^{N}\left|J_{k}\left(r e^{i \theta}\right)-J_{n}\left(r e^{i \theta}\right)\right|^{-1} .
$$

For $p<1 / N,\left(\prod_{k=1 ; k \neq n}^{N}\left|J_{k}\left(r e^{i \theta}\right)-J_{n}\left(r e^{i \theta}\right)\right|\right)^{-1}$ is in $L^{p}$ so $C_{n}$ is in $H^{p}$. Since the boundary values of $C_{n}$ are in $L^{\infty}$, it follows that $C_{n}$ is actually in $H^{\infty}$.

CoRollary. Let $\phi$ be a covering map of $D$ onto $\Omega$, let $I_{1}, \ldots, I_{N}$ be distinct elements of the covering group, and let $C_{1}, \ldots, C_{N}$ be functions analytic on $D$. The operator, $S$, on $H^{2}$ defined by

$$
(S g)(\alpha)=\sum_{k=1}^{N} C_{k}(\alpha) g\left(I_{k}(\alpha)\right)
$$

is bounded and in $\left\{T_{\phi}\right\}^{\prime}$ if and only if the functions $C_{1}, \ldots, C_{N}$ are bounded on the disk.

Proof. This result is obvious from Theorems 7 and 8 above.

We observe that in the situation in the corollary, the operator $S$ takes bounded functions to bounded functions.

We close this section with a comment on another of the questions raised by Deddens and Wong [4]. Abrahamse and Ball [2] have recently shown that $\left\{T_{\psi_{1}}\right\}^{\prime} \cap\left\{T_{\psi_{2}}\right\}^{\prime}$ need not be the commutant of an analytic Toeplitz operator whose symbol is inner even if $\psi_{1}$ and $\psi_{2}$ are inner. We give a more elementary example of this phenomenon which is in the spirit of this work. 
Let $A$ be the annulus $A=\left\{z\left|\frac{1}{2}<\right| z \mid<2\right\}$, and $\phi$ a covering map of $D$ onto $A$. Let $h_{1}(z)=z+1 / z$ and let $h_{2}(z)=z-1 / z$. Now $h_{1}$ and $h_{2}$ map $A$ two-to-one onto ellipses. Let $g_{1}$ and $g_{2}$ be univalent maps of these ellipses onto $D$. We then define $\psi_{1}$ and $\psi_{2}$ by $\psi_{j}=g_{j} \circ h_{j} \circ \phi$. Clearly $\psi_{1}$ and $\psi_{2}$ are inner functions. Moreover, for $\beta$ in $A, h_{1}^{-1}\left(h_{1}(\beta)\right)=\{\beta, 1 / \beta\}$ and $h_{2}^{-1}\left(h_{2}(\beta)\right)=\{\beta,-1 / \beta\}$ so it follows that for every $\alpha$ in $D$,

$$
\psi_{1}^{-1}\left(\psi_{1}(\alpha)\right) \cap \psi_{2}^{-1}\left(\psi_{2}(\alpha)\right)=\phi^{-1}(\phi(\alpha)) .
$$

Therefore, $\left\{T_{\psi_{1}}\right\}^{\prime} \cap\left\{T_{\psi_{2}}\right\}^{\prime}=\left\{T_{\phi}\right\}^{\prime}$, which we have seen is not the same as the commutant of a Toeplitz operator whose symbol is inner.

5. Compacts in the commutant. In this section we examine the question of compact operators in the commutant of an analytic Toeplitz operator. This question is distantly related to Lomonosov's theorem on invariant subspaces [7]. Lomonosov proves that if $A, B$, and $C$, not multiples of the identity, are such that $C$ is compact and $B$ commutes with both $A$ and $C$, then $A$ has a nontrivial invariant subspace. If every operator $A$ satisfies the hypotheses of this theorem, then the invariant subspace problem is solved. It seems likely that $T_{z}$ does not satisfy the hypotheses. The question "Does $T_{z}$ satisfy the hypotheses of this theorem?" is just the question "Is there a nonconstant $f$ in $H^{\infty}$ such that $T_{f}$ commutes with a nonzero compact operator?" [4].

The two theorems of this section give conditions on an $H^{\infty}$ function, $f$, which are sufficient to show that $T_{f}$ does not commute with any nonzero compact operators. We begin by proving two lemmas.

LEMMA A. If $f$ is a nonconstant function in $H^{\infty}$ and $R$ is a compact operator which commutes with $T_{f}$, then $R$ is quasi-nilpotent.

Proof. Let $\lambda$ be a nonzero complex number, and let $M_{\lambda}$ be the kernel of $R-\lambda$. Since $R$ is compact and commutes with $T_{f}, M_{\lambda}$ must be finite dimensional and invariant for $T_{f}$. But since $f$ is nonconstant, this means $M_{\lambda}$ is the zero subspace. That is, $\lambda$ is not in the spectrum of $R$.

For $\alpha$ a point of $D$, we let $b_{\alpha}(z)=(z-\alpha) /(1-\bar{\alpha} z)$.

Lemma B. If $f$ is in $H^{\infty}, S$ commutes with $T_{f}$ and $S^{*} K_{\alpha}=0$, then $T_{b_{\alpha}}^{*} S$ commutes with $T_{f}$.

Proof. The condition $S^{*} K_{\alpha}=0$ means $S H^{2} \subset b_{\alpha} H^{2}$. Given $h$ in $H^{2}$, let $S h=b_{\alpha} g$. We have

$$
T_{b_{\alpha}}^{*} S T_{f} h=T_{b_{\alpha}}^{*} T_{f} S h=T_{b_{\alpha}}^{*} f b_{\alpha} g=f g=T_{f} g=T_{f} T_{b_{\alpha}}^{*} S h .
$$

Since $h$ was arbitrary we have $\left(T_{b_{\alpha}}^{*} S\right) T_{f}=T_{f}\left(T_{b_{a}}^{*} S\right)$.

Definition. Let $f$ be in $H^{\infty}$ and let $\alpha$ be a point of $D$. We say $f$ has property $K$.T. at $\alpha$ if whenever $A^{*} K_{\alpha}=K_{\beta}$ for some $\beta$ in $D$ and $A$ in $\left\{T_{f}\right\}^{\prime}$, then there is an operator $B$ in $\left\{T_{f}\right\}^{\prime}$ so that $B^{*} K_{\beta}=K_{\alpha}$. 
The property K.T. at $\alpha$ is a transitivity property of $\left\{T_{f}^{*}\right\}^{\prime}$ acting on the set of kernel functions $K_{\beta}$ with $f(\beta)=f(\alpha)$.

THEOREM 9. Suppose $f$ is in $H^{\infty}$ and $\alpha$ is a point of $D$ so that $\operatorname{Inn}(f-f(\alpha))$ is a Blaschke product with distinct zeroes. If $f$ has property K.T. at $\alpha$, then $T_{f}$ does not commute with any nonzero compact operators.

Proof. We observe that $(f-f(\alpha)) H^{2 \perp}$ is spanned by $\left\{K_{\beta} \mid f(\beta)=f(\alpha)\right\}$. Let $R$ be a compact operator which commutes with $T_{f}$. If $R^{*} K_{\alpha} \neq 0$, we can find $\delta$ in $D, f(\delta)=f(\alpha)$, such that $R^{*} K_{\alpha}$ is not in the span of $\left\{K_{\beta} \mid f(\beta)=\right.$ $f(\alpha), \beta \neq \delta\}$. For such a $\delta$, we can write $R^{*} K_{\alpha}=c K_{\delta}+h_{\delta}$ where $c \neq 0$ and $h_{\delta}$ is in the span $\left\{K_{\beta} \mid f(\beta)=f(\alpha), \beta \neq \delta\right\}$. Since $\operatorname{Inn}(f-f(\alpha))$ has distinct zeroes, $g(z)=(f(z)-f(\alpha)) /(z-\delta)$ does not vanish at $\delta$. Let $\tilde{R}=$ $(\bar{c} g(\delta))^{-1} R T_{g}$. Clearly $\tilde{R}$ commutes with $T_{f}$ and

$$
\tilde{R}^{*} K_{\alpha}=(c \overline{g(\delta)})^{-1} T_{g}^{*} R^{*} K_{\alpha}=K_{\delta} .
$$

Since $f$ has property K.T. at $\alpha$, there is an operator $B$ in $\left\{T_{f}\right\}^{\prime}$ such that $B^{*} K_{\delta}=K_{\alpha}$. Therefore, $\tilde{R} B$ is compact, commutes with $T_{f}$, and $(\tilde{R} B)^{*} K_{\alpha}=$ $K_{\alpha}$. That is, $(\tilde{R} B)^{*}$ has 1 as an eigenvalue, and 1 is in the spectrum of $\tilde{R} B$. This contradicts the result of Lemma $\mathrm{A}$, that $\tilde{R} B$ is quasi-nilpotent. We conclude then that actually $R^{*} K_{\alpha}=0$.

We have shown that the adjoint of a compact operator which commutes with $T_{f}$ has $K_{\alpha}$ in its kernel. From Lemma B we see that $R^{*} K_{\alpha}=0$ implies $T_{b_{a}}^{*} R$ commutes with $T_{f}$. Since $T_{b_{a}}^{*} R$ is also compact, we have

$$
R^{*}\left(b_{\alpha} K_{\alpha}\right)=R^{*} T_{b_{\alpha}} K_{\alpha}=\left(T_{b_{\alpha}}^{*} R\right)^{*} K_{\alpha}=0 .
$$

By induction we see that $R^{*}\left(b_{\alpha}^{n} K_{\alpha}\right)=0$ for $n=0,1,2,3, \ldots$ Since the set $\left\{b_{\alpha}^{n} K_{\alpha}\right\}_{n=0}^{\infty}$ spans $H^{2}$, we conclude $R^{*}=0$ and $R=0$.

COROLlary. If $f$ is ancestral, then $T_{f}$ does not commute with any nonzero compact operators.

Proof. By Rudin's Theorem ( $\$ 3)$, there is a point $\alpha$ in $D$ such that $\operatorname{Inn}(f-f(\alpha))$ is a Blaschke product with distinct zeroes. We prove that for this $\alpha$, since $f$ is ancestral, $f$ has the property K.T. at $\alpha$.

Suppose for some $A$ in $\left\{T_{f}\right\}^{\prime}, A^{*} K_{\alpha}=K_{\beta}$. Since $f$ is ancestral, the closed span of $\left\{C^{*} K_{\beta} \mid C \in\left\{T_{f}\right\}^{\prime}\right\}$ is all of $(f-f(\beta)) H^{2 \perp}=(f-f(\alpha)) H^{2 \perp}$. So for some $C$ in $\left\{T_{f}\right\}^{\prime}, C^{*} K_{\beta}=c K_{\alpha}+h$ where $c \neq 0$ and $h$ is in the closed span of $\left\{K_{\delta} \mid f(\delta)=f(\alpha), \delta \neq \alpha\right\}$. Since the zeroes of $\operatorname{Inn}(f-f(\alpha))$ are distinct, $g(z)=(f(z)-f(\alpha)) /(z-\alpha)$ does not vanish at $\alpha$. Let $B=(\bar{c} g(\alpha))^{-1} C T_{g}$. Then $B$ is in $\left\{T_{f}\right\}^{\prime}$ and $B^{*} K_{\beta}=K_{\alpha}$.

Thus $f$ has the property K.T. at $\alpha$, and we apply the theorem to obtain the conclusion.

It should be noted that this corollary applies to inner functions and to 
covering maps; indeed, the theorem applies to all $H^{\infty}$ functions, the commutant of whose Toeplitz operators is known. It is not known whether all $H^{\infty}$ functions satisfy the property K.T. for some $\alpha$ in $D$.

Up to this point, the results and proofs have been essentially related to the properties of analytic functions in the disk with very little emphasis on their boundary values. The next theorem changes this emphasis: the essential part of the argument is based on the boundary behavior of the function.

THEOREM 10. Let $f$ be a nonconstant function in $H^{\infty}$. If there is a set $F$ such that:

(i) $F$ is closed and connected,

(ii) $F \cap f(D)=\varnothing$, and

(iii) the set $\left\{e^{i t} \in \partial D \mid \lim _{r \rightarrow 1} f\left(r e^{i t}\right) \in F\right\}$ has positive measure, then no nonzero compact operator commutes with $T_{f}$.

The set $F$ is a set of boundary values of $f$ which are not values of $f$ restricted to $D$.

PRoof. Let $\tilde{E}=\left\{e^{i t} \mid \lim _{r \rightarrow 1} f\left(r e^{i t}\right) \in F\right\}$. Since $f$ is nonconstant and $\tilde{E}$ has positive measure, the set $F$ contains more than one point. Let $\Omega$ be the component of $\overline{\mathbf{C}} \backslash F$ containing $f(D)$. Since $F$ is closed and connected, $\partial \Omega \subset F$ and $\Omega$ is simply connected. Let $\psi$ be a Riemann map of $\Omega$ onto $D$, and $g=\psi \circ f$. It follows from the corollary to the Fundamental Lemma that $\left\{T_{f}\right\}^{\prime}=\left\{T_{g}\right\}^{\prime}$. We will show $T_{g}$ does not commute with any nonzero compact operators.

We first show that the set $E=\left\{e^{i t}|| \lim _{r \rightarrow 1} g\left(r e^{i t}\right) \mid=1\right\}$ has positive measure. Suppose for some $\theta$ in $[0,2 \pi), \lim _{r \rightarrow 1} g\left(r e^{i \theta}\right)=\alpha$, and $|\alpha|<1$. Since $\psi^{-1}(g(z))=f(z)$ for all $z$ in $D$ and since $\psi^{-1}$ is analytic near $\alpha$, we have

$$
\lim _{r \rightarrow 1} f\left(r e^{i \theta}\right)=\lim _{r \rightarrow 1} \psi^{-1}\left(g\left(r e^{i \theta}\right)\right)=\psi^{-1}(\alpha),
$$

which is not in $F$. That is, $E$ contains $\tilde{E} \cap\left\{e^{i t} \mid \lim _{r \rightarrow 1} g\left(r e^{i t}\right)\right.$ exists $\}$, so $E$ has positive measure.

Now let $R$ be a compact operator which commutes with $T_{g}$. For any integer $k, k>0$, the sequence $\left\{z^{k} g^{n}\right\}_{n=1}^{\infty}$ converges weakly to zero in $H^{2}$. (For any $\alpha$ in $D$, since $|g(\alpha)|<1$, any limit point of the sequence has value 0 at $\alpha$. Since the only limit point is $\mathbf{0}$ and since the sequence is contained in the unit ball which is weakly compact, the sequence converges weakly to 0 .)

It follows that

$$
\lim _{n \rightarrow \infty}\left\|g^{n} R z^{k}\right\|=\lim _{n \rightarrow \infty}\left\|T_{g}^{n} R z^{k}\right\|=\lim _{n \rightarrow \infty}\left\|R z^{k} g^{n}\right\|=0 .
$$

But since $|g|=1$ on $E$, we have 


$$
\left\|g^{n} R z^{k}\right\|^{2}=\frac{1}{2 \pi} \int_{0}^{2 \pi}\left|g^{n}(t)\right|^{2}\left|\left(R z^{k}\right)(t)\right|^{2} d t \geqslant \frac{1}{2 \pi} \int_{E}\left|\left(R z^{k}\right)(t)\right|^{2} d t .
$$

Therefore $R z^{k}$ has boundary value 0 on $E$, which is a set of positive measure, which means $R z^{k}=0$. Since $k$ was arbitrary, $R=0$.

Many interesting functions satisfy the hypotheses of this theorem, for example inner functions or functions continuously differentiable on the unit circle. On the other hand, not all functions satisfy the hypotheses. For example, suppose $\Gamma$ is a spiral in $D$ which approaches the unit circle asymptotically, $\Gamma \cap(-\Gamma)=\varnothing$, and $\psi$ is a univalent map of $D$ onto $D \backslash \Gamma$. Then $\psi^{2}$ does not satisfy the hypotheses of Theorem 10 . Indeed, the best candidate for $F$ is $\partial D$, but the set $E$ corresponding to $\partial D$ is a single point! (The final corollary of $\S 3$ shows that $\left\{T_{\psi^{2}}\right\}^{\prime}=\left\{T_{z}\right\}^{\prime}$ so the conclusion is still valid.)

It would be interesting to know whether the hypotheses of either Theorem 9 or Theorem 10 apply to all functions continuous on the unit circle.

6. Comments and questions. For the most part, this paper has dealt with two questions:

(i) identify functions whose Toeplitz operators have "nice" commutants; and

(ii) given an analytic Toeplitz operator $T_{f}$, find a "nice" function $\phi$ so that $\left\{T_{f}\right\}^{\prime}=\left\{T_{\phi}\right\}^{\prime}$.

The notion of $H^{2}$-ancestral functions was introduced in $\$ 1$ because these functions are in some sense "nice". The class of $H^{2}$-ancestral functions gives rise to a class of Toeplitz operators with distinct commutants which behave well with respect to composition of the symbol.

Question I. Is there, for each $f$ in $H^{\infty}$, an $H^{2}$-ancestral function $\phi$ such that $\left\{T_{f}\right\}^{\prime}=\left\{T_{\phi}\right\}^{\prime}$ ?

We have seen that inner functions and covering maps are $H^{2}$-ancestral, but these functions do not exhaust the class. For example, suppose

$$
\Omega=D \backslash\left\{z \mid z \text { is real and } \frac{1}{2}<|z|<\frac{3}{4}\right\} .
$$

Let $\phi$ be a covering map of $D$ onto $\Omega$ and let $f=\phi^{2}$. It is easy to see that $f$ is $H^{2}$-ancestral, that $f$ is not a covering map, and that $\left\{T_{f}\right\}^{\prime} \neq\left\{T_{\psi}\right\}^{\prime}$ if $\psi$ is an inner function. Especially if the answer to Question I is yes, we would like to have a better description of $H^{2}$-ancestral functions.

Question II. Is there a function theoretic description of the class of $H^{2}$-ancestral functions?

Theorem 4 gives conditions on a family $\mathscr{F}$ of $H^{\infty}$ functions which imply that there is a function $\phi$ such that $\left\{T_{\phi}\right\}^{\prime}=\bigcap_{h \in \mathscr{F}}\left\{T_{h}\right\}^{\prime}$. Perhaps the conditions on $\mathscr{F}$ are unnecessary.

Question III. Given an arbitrary family $\mathscr{F}$ of $H^{\infty}$ functions, is there a 
function $\phi$ such that $\left\{T_{\phi}\right\}^{\prime}=\bigcap_{h \in \mathcal{F}}\left\{T_{h}\right\}^{\prime}$ ?

It is hoped that the results and methods of this paper will be helpful in studying more general subnormal operators. The extent to which this hope can be realized depends on the extent to which analytic Toeplitz operators are typical subnormal operators. They should at least be typical of operators which can be realized as multiplication by analytic functions in a Hilbert space of analytic functions. In their paper The commutants of certain Hilbert space operators [11], Shields and Wallen identified certain Hilbert spaces of analytic functions in which the operators which commute with multiplication by $z$ are just multiplication by other $H^{\infty}$ functions. Many of the techniques used here to study analytic Toeplitz operators can be used to study the analytic multiplication operators on these spaces. If $\mathcal{H C}$ is a Hilbert space of analytic functions on $D$ as considered by Shields and Wallen, and $M_{f}$ is the operator on $\mathcal{H}$ of multiplication by $f$, then $\left\{g \in H^{\infty} \mid M_{g} \in\left\{M_{f}\right\}^{\prime \prime}\right\}$ is a subalgebra of $H^{\infty}$ which we will denote by $\{f\}_{\mathcal{K}}^{\prime \prime}$.

Question IV. Given $f$ in $H^{\infty}$, is the subalgebra $\{f\}_{\mathcal{C}}^{\prime \prime}$ the same for each of the Hilbert spaces $\mathcal{H}$ ?

Clearly, for some $f$ in $H^{\infty}$, the answer to Question IV is yes. Indeed, Shields and Wallen show that $\{z\}_{\mathcal{C}}^{\prime \prime}=H^{\infty}$ for these spaces, and the techniques from $\$ 2$ show that if $\phi$ is a finite Blaschke product then, for each of these spaces, $\{\phi\}_{\mathcal{C}}^{\prime \prime}=\left\{f \in H^{\infty} \mid f=g \circ \phi\right.$ for some $\left.g \in H^{\infty}\right\}$.

For these spaces, we can define the notion of $\mathcal{K}$-ancestral function analogous to the notion of $\mathrm{H}^{2}$-ancestral function.

Question V. Is the set of $\mathcal{K}$-ancestral functions the same for each of the Hilbert spaces $\mathcal{I C}$ ?

Question V is related to Question II above. We would expect that if there is a simple function theoretic description of $H^{2}$-ancestral functions, then the same description would apply to $\mathcal{K}$-ancestral functions.

\section{BIBLIOGRAPHY}

1. M. B. Abrahamse, Analytic Toeplitz operators with automorphic symbol, Proc. Amer. Math. Soc. 52 (1975), 297-302. MR 53 \#8951.

2. M. B. Abrahamse and J. A. Ball, Analytic Toeplitz operators with automorphic symbol. II, Proc. Amer. Math. Soc. 59 (1976), 323-328.

3. I. N. Baker, J. A. Deddens and J. L. Ullman, $A$ theorem on entire functions with applications to Toeplitz operators, Duke Math. J. 41 (1974), 739-745. MR 50 \#7523.

4. J. A. Deddens and T. K. Wong, The commutant of analytic Toeplitz operators, Trans. Amer. Math. Soc. 184 (1973), 261-273. MR 48 \#2819.

5. K. Hoffman, Banach spaces of analytic functions, Prentice-Hall, Englewood Cliffs, N.J., 1962. MR 24 \#A2844.

6. E. A. Nordgren, Composition operators, Canad. J. Math. 20 (1968), 442-449. MR 36 \#6961.

7. C. Pearcy and A. L. Shields, $A$ survey of the Lomonosov technique in the theory of invariant subspaces, Math. Surveys, No. 13, Amer. Math. Soc., Providence, R.I., 1974, pp. 219-229. MR 50 \#8113. 
8. W. Rudin, A generalization of a theorem of Frostman, Math. Scand. 21 (1967), 136-143 (1968). MR 38 \#3463.

9. J. V. Ryff, Subordinate $H^{p}$ functions, Duke Math. J. 33 (1966), 347-354. MR 33 \#289.

10. H. S. Shapiro and A. L. Shields, On some interpolation problems for analytic functions, Amer. J. Math. 83 (1961), 513-532. MR 24 \#A3280.

11. A. L. Shields and L. J. Wallen, The commutants of certain Hilbert space operators, Indiana Univ. Math. J. 20 (1970/71), 777-788. MR 44 \#4558.

12. J. E. Thomson, Intersections of commutants of analytic Toeplitz operators, Proc. Amer. Math. Soc. 52 (1975), 305-310. MR 53 \#3765.

13. , The commutants of certain analytic Toeplitz operators, Proc. Amer. Math. Soc. 54 (1976), 165-169. MR 52 \#8993.

14. __ The commutant of a class of analytic Toeplitz operators, Amer. J. Math. 99 (1977), $522-529$.

15. . The commutant of a class of analytic Toeplitz operators. II, Indiana Univ. Math. J. 25 (1976), 793-800.

16. W. A. Veech, $A$ second course in complex analysis, Benjamin, New York, 1967. MR 36 \#3955.

17. E. L. Stout, Bounded holomorphic functions on finite Riemann surfaces, Trans. Amer. Math. Soc. 120 (1965), 255-285. MR 32 \#1358.

Department of Mathematics, University of California, Berkeley, California 94720

Current address: Department of Mathematics, University of Illinois, Urbana, Illinois 61801 